\title{
TIME-CHANGED PROCESSES GOVERNED BY SPACE-TIME FRACTIONAL TELEGRAPH EQUATIONS
}

\author{
MIRKO D'OVIDIO, ENZO ORSINGHER, AND BRUNO TOALDO
}

\begin{abstract}
In this work we construct compositions of vector processes of the form $\boldsymbol{S}_{n}^{2 \beta}\left(c^{2} \mathcal{L}^{\nu}(t)\right), t>0, \nu \in\left(0, \frac{1}{2}\right], \beta \in(0,1], n \in \mathbb{N}$, whose distribution is related to space-time fractional $n$-dimensional telegraph equations. We present within a unifying framework the pde connections of $n$-dimensional isotropic stable processes $\boldsymbol{S}_{n}^{2 \beta}$ whose random time is represented by the inverse $\mathcal{L}^{\nu}(t)$, $t>0$, of the superposition of independent positively-skewed stable processes, $\mathcal{H}^{\nu}(t)=H_{1}^{2 \nu}(t)+(2 \lambda)^{\frac{1}{\nu}} H_{2}^{\nu}(t), t>0,\left(H_{1}^{2 \nu}, H_{2}^{\nu}\right.$, independent stable subordinators). As special cases for $n=1, \nu=\frac{1}{2}$ and $\beta=1$ we examine the telegraph process $T$ at Brownian time $|B|$ (Orsingher and Beghin [13]) and establish the equality in distribution $B\left(c^{2} \mathcal{L}^{\frac{1}{2}}(t)\right) \stackrel{\text { law }}{=} T(|B(t)|), t>0$. Furthermore the iterated Brownian motion (Allouba and Zheng [2]) and the two-dimensional motion at finite velocity with a random time are investigated. For all these processes we present their counterparts as Brownian motion at delayed stabledistributed time.
\end{abstract}

\section{Contents}

1. Introduction and preliminaries

1.1. Introduction

1.2. Notations

1.3. Preliminaries

2. Sum of stable subordinators, $\mathcal{H}^{\nu}(t)=H_{1}^{2 \nu}(t)+(2 \lambda)^{\frac{1}{\nu}} H_{2}^{\nu}(t)$

2.1. The inverse process $\mathcal{L}^{\nu}(t) \quad 11$

3. $n$-dimensional stable laws and fractional Laplacian $\quad 15$

4. Space-time fractional telegraph equation $\quad 17$

$\begin{array}{ll}\text { 4.1. The case } \nu=\frac{1}{2} \text {, subordinator with drift } & 19\end{array}$

4.2. The case $\nu=\frac{1}{3}$, convolutions of Airy functions 24

$\begin{array}{ll}\text { 4.3. The planar case } & 27\end{array}$

References 33

\section{INTRODUCTION AND PRELIMINARIES}

1.1. Introduction. The study of the interplay between fractional equations and stochastic processes has began in the middle of the Eighties with the analysis of

Date: July 25, 2018.

2000 Mathematics Subject Classification. 60G51, 60G52, 35C05.

Key words and phrases. Riemann-Liouville fractional calculus, Telegraph processes, Stable positively skewed r.v.'s, Subordinators, Fractional Laplacian, Mittag-Leffler functions, Timechanged processes, Airy functions. 
simple time-fractional diffusion equations (see Fujita [9] for a rigorous work on this field, or more recently Allouba and Nane [1], where the compositions of Brownian sheets with Brownian motions are considered). In some papers the connection between fractional diffusion equations and stable processes is explored (see, for example, Orsingher and Beghin [16]; Zolotarev [20]). The iterated Brownian motion has distribution satisfying the following fractional equation

$$
\frac{\partial^{\frac{1}{2}}}{\partial t^{\frac{1}{2}}} u(x, t)=\frac{1}{2^{\frac{3}{2}}} \frac{\partial^{2}}{\partial x^{2}} u(x, t), \quad x \in \mathbb{R}, t>0,
$$

(see for example Allouba and Zheng [2]) and also the fourth-order equation

$$
\frac{\partial}{\partial t} u(x, t)=\frac{1}{2^{3}} \frac{\partial^{4}}{\partial x^{4}} u(x, t)+\frac{1}{2 \sqrt{2 \pi t}} \frac{d^{2}}{d x^{2}} \delta(x), \quad x \in \mathbb{R}, t>0,
$$

see DeBlassie [6] (also for an interpretation of the iterated Brownian motion to model the motion of a gas in a crack).

When the fractional equation has a telegraph structure, with more than one time-fractional derivative involved, that is for $\nu \in(0,1]$

$$
\left(\frac{\partial^{2 \nu}}{\partial t^{2 \nu}}+2 \lambda \frac{\partial^{\nu}}{\partial t^{\nu}}\right) u(x, t)=c^{2} \frac{\partial^{2}}{\partial x^{2}} u(x, t), \quad x \in \mathbb{R}, t>0, \lambda>0, c>0,
$$

the relationship of its solution with the time-changed telegraph processes is examined and established in Orsingher and Beghin [13] . The space-fractional telegraph equation (with M. Riesz space derivatives) has been considered in Orsingher and Zhao [14], while the connection between space-fractional equations and asymmetric stable processes has been established in Feller [7].

Fractional telegraph equations from the analytic point of view have been studied by many authors (see Saxena, Mathai and Haubold [18] for equations with $n$ time derivatives). For their solutions have been worked out also numerical techniques (see, for example, Momani [12]). Telegraph equations have an extraordinary importance in electrodynamics (the scalar Maxwell equations are of this type), in the theory of damped vibrations and in probability because they are connected with finite velocity random motions.

In this paper we consider various types of processes obtained by composing symmetric stable processes $\boldsymbol{S}_{n}^{2 \beta}(t), t>0,0<\beta \leq 1$, with the inverse of the sum of two independent stable subordinators (instead of one as in ? ]) say $\mathcal{L}^{\nu}(t), t>0$, $0<\nu \leq \frac{1}{2}$. These time-changed processes, $\boldsymbol{W}_{n}(t)=\boldsymbol{S}_{n}^{2 \beta}\left(c^{2} \mathcal{L}^{\nu}(t)\right), t>0$, have distributions, $w_{\nu}^{\beta}(\boldsymbol{x}, t), \boldsymbol{x} \in \mathbb{R}^{n}, t>0$, which satisfy telegraph-type space-time fractional equations of the form

$$
\left(\frac{{ }^{C} \partial^{2 \nu}}{\partial t^{2 \nu}}+2 \lambda \frac{{ }^{C} \partial^{\nu}}{\partial t^{\nu}}\right) w_{\nu}^{\beta}(\boldsymbol{x}, t)=-c^{2}(-\Delta)^{\beta} w_{\nu}^{\beta}(\boldsymbol{x}, t), \quad \boldsymbol{x} \in \mathbb{R}^{n}, t>0, c>0, \lambda>0,
$$

where $0<\beta \leq 1,0<\nu \leq \frac{1}{2}$, subject to the initial condition

$$
w_{\nu}^{\beta}(\boldsymbol{x}, 0)=\delta(\boldsymbol{x}) .
$$

The fractional Laplacian $(-\Delta)^{\beta}$, appearing in (1.4), is defined and analyzed in Section 3 below. The fractional derivatives appearing in (1.4) are meant in the Dzerbayshan-Caputo sense, that is, for an absolutely continuous function $f \in$ 
$L^{1}(\mathbb{R})$ (for fractional calculus consult Kilbas, Srivastava and Trujillo [11]),

$$
\frac{{ }^{C} \partial^{\nu}}{\partial t^{\nu}} f(t)=\frac{1}{\Gamma(m-\nu)} \int_{0}^{t} \frac{\frac{d^{m}}{d s^{m}} f(s)}{(t-s)^{\nu+1-m}} d s, \quad m-1<\nu<m, m \in \mathbb{N} .
$$

Equation (1.4) includes as particular cases all fractional equations studied so far (including diffusion equations) and also the main equations of mathematical physics as limit cases. Thus the distribution of the composed process $\boldsymbol{S}_{n}^{2 \beta}\left(\mathcal{L}^{\nu}(t)\right), t>0$, represents the fundamental solution of the most general $n$-dimensional time-space fractional telegraph equation. We give the general Fourier transform of the solution to (1.4) with initial condition (1.5) as

$$
\begin{aligned}
& \mathbb{E} e^{i \boldsymbol{\xi} \cdot \boldsymbol{S}_{n}^{2 \beta}\left(c^{2} \iota^{\nu}(t)\right)=} \\
= & \frac{1}{2}\left[\left(1+\frac{\lambda}{\sqrt{\lambda^{2}-c^{2}\|\boldsymbol{\xi}\|^{2 \beta}}}\right) E_{\nu, 1}\left(r_{1} t^{\nu}\right)+\left(1-\frac{\lambda}{\sqrt{\lambda^{2}-c^{2}\|\boldsymbol{\xi}\|^{2 \beta}}}\right) E_{\nu, 1}\left(r_{2} t^{\nu}\right)\right],
\end{aligned}
$$

where

$$
r_{1}=-\lambda+\sqrt{\lambda^{2}-c^{2}\|\boldsymbol{\xi}\|^{2 \beta}}, \quad r_{2}=-\lambda-\sqrt{\lambda^{2}-c^{2}\|\boldsymbol{\xi}\|^{2 \beta}} .
$$

and

$$
E_{\nu, \psi}(x)=\sum_{k=0}^{\infty} \frac{x^{k}}{\Gamma(\nu k+\psi)}, \quad \nu, \psi>0,
$$

is the two-parameters Mittag-Leffler function (see, for example, Haubold, Mathai and Saxena [10] for a general overview on the Mittag-Leffler functions). Our result therefore includes all previous results in a unique framework and sheds an additional insight into the literature in this field.

An important role in our analysis is played by the time change based on the process $\mathcal{L}^{\nu}(t), t>0$. We consider first the sum of two independent positively skewed stable r.v.'s $H_{1}^{2 \nu}(t)$ and $H_{2}^{\nu}(t), t>0,0<\nu \leq \frac{1}{2}$,

$$
\mathcal{H}^{\nu}(t)=H_{1}^{2 \nu}(t)+(2 \lambda)^{\frac{1}{\nu}} H_{2}^{\nu}(t), \quad t>0,
$$

whose distribution $h_{\nu}(x, t)$ is governed by the space fractional equation

$$
\frac{\partial}{\partial t} \hbar_{\nu}(x, t)=-\left(\frac{\partial^{2 \nu}}{\partial x^{2 \nu}}+2 \lambda \frac{\partial^{\nu}}{\partial x^{\nu}}\right) \hbar_{\nu}(x, t), \quad x \geq 0, t>0,0<\nu \leq \frac{1}{2} .
$$

In (1.11) the fractional derivatives must be meant in the Riemann-Liouville sense which, for a function $f \in L^{1}(\mathbb{R})$, is defined as

$$
\frac{\partial^{\nu}}{\partial x^{\nu}} f(x)=\frac{1}{\Gamma(m-\nu)} \frac{d^{m}}{d x^{m}} \int_{0}^{x} \frac{f(s)}{(x-s)^{\nu+1-m}} d s, \quad m-1<\nu<m, m \in \mathbb{N} .
$$

We then take the inverse $\mathcal{L}^{\nu}(t), t>0$, to the process $\mathcal{H}^{\nu}(t), t>0$, defined as

$$
\mathcal{L}^{\nu}(t)=\inf \left\{s>0: H_{1}^{2 \nu}(s)+(2 \lambda)^{\frac{1}{\nu}} H_{2}^{\nu}(s) \geq t\right\}, \quad t>0,
$$

whose distribution is related to that of $\mathcal{H}^{\nu}(t), t>0$, by means of the formula

$$
\operatorname{Pr}\left\{\mathcal{L}^{\nu}(t)<x\right\}=\operatorname{Pr}\left\{\mathcal{H}^{\nu}(x)>t\right\} .
$$


The distribution $\zeta_{\nu}(x, t)$ of $\mathcal{L}^{\nu}(t), t>0$, satisfies the time-fractional telegraph equation

$$
\left(\frac{\partial^{2 \nu}}{\partial t^{2 \nu}}+2 \lambda \frac{\partial^{\nu}}{\partial t^{\nu}}\right) c_{\nu}(x, t)=-\frac{\partial}{\partial x} c_{\nu}(x, t), \quad x \geq 0, t>0,0<\nu \leq \frac{1}{2},
$$

where the fractional derivatives appearing in (1.15) are again in the RiemannLiouville sense. We are able to give explicit forms of the Laplace transforms of $\zeta_{\nu}(x, t)$ and $\zeta_{\nu}(x, t)$ in terms of Mittag-Leffler functions for all values of $0<\nu \leq \frac{1}{2}$. For example, for the distribution $\zeta_{\nu}(x, t)$ of $\mathcal{L}^{\nu}(t)$ we have that, for $\gamma<\lambda^{2}$,

$$
\begin{aligned}
& \int_{0}^{\infty} e^{-\gamma x} c_{\nu}(x, t) d x= \\
= & \frac{1}{2}\left[\left(1+\frac{\lambda}{\sqrt{\lambda^{2}-\gamma}}\right) E_{\nu, 1}\left(r_{1} t^{\nu}\right)+\left(1-\frac{\lambda}{\sqrt{\lambda^{2}-\gamma}}\right) E_{\nu, 1}\left(r_{2} t^{\nu}\right)\right],
\end{aligned}
$$

where

$$
r_{1}=-\lambda+\sqrt{\lambda^{2}-\gamma}, \quad r_{2}=-\lambda-\sqrt{\lambda^{2}-\gamma} .
$$

The distribution $\epsilon_{\nu}(x, t)$ of $\mathcal{L}^{\nu}(t), t>0$, has the general form

$$
\zeta_{\nu}(x, t)=\int_{0}^{t} l_{2 \nu}(x, s) h_{\nu}(t-s, 2 \lambda x) d s+2 \lambda \int_{0}^{t} l_{\nu}(2 \lambda x, s) h_{2 \nu}(t-s, x) d s,
$$

where the distributions of $H^{2 \nu}, H^{\nu}$, and that of their inverse processes $L^{2 \nu}$ and $L^{\nu}$ appear. For our analysis it is relevant to obtain the distributions of $\mathcal{H}^{\frac{1}{2}}(t)$, $t>0$, and $L^{\frac{1}{2}}(t), t>0$. We also obtain explicitely the distributions of $H^{\frac{1}{3}}(t)$ and $H^{\frac{2}{3}}(t), t>0$, and also of their inverses $L^{\frac{1}{3}}(t)$ and $L^{\frac{2}{3}}(t), t>0$, in terms of Airy functions. By means of the convolutions of these distributions we arrive at the following cumbersome density of the random time $\mathcal{L}^{\frac{1}{3}}(t), t>0$,

$$
\begin{aligned}
\operatorname{Pr}\left\{L^{\frac{1}{3}}(t) \in d x\right\}= & \frac{2 \lambda}{\sqrt{\pi}} \int_{0}^{t} d s \int_{0}^{\infty} d w e^{-w} w^{-\frac{1}{6}} \operatorname{Ai}\left(-x \sqrt[3]{\frac{2^{2} w}{3(t-s)^{2}}}\right) \operatorname{Ai}\left(\frac{2 \lambda x}{\sqrt[3]{3 s}}\right) \cdot \\
& \cdot \frac{3}{\sqrt[3]{3 s}} \sqrt[3]{\frac{2^{2}}{3(t-s)^{2}}}\left[\frac{x}{2 s}+\frac{s}{t-s}\right] d x
\end{aligned}
$$

For $n=1, \beta=1$ and $\nu=1$ in (1.4), we get the telegraph equation which is satisfied by the distribution of the one-dimensional telegraph process

$$
T(t)=V(0) \int_{0}^{t}(-1)^{N(s)} d s, \quad t>0,
$$

where $N(t), t>0$ is an homogeneous Poisson process, with parameter $\lambda>0$, independent from the symmetric r.v. $V(0)$ (with values $\pm c$ ). Properties of this process (including first-passage time distributions) are studied in Foong and Kanno [8] and a telegraph process with random velocities has been recently considered by Stadje and Zacks [19].

For $n=1, \beta=1$ and $\nu=\frac{1}{2}$ the special equation

$$
\left\{\begin{array}{l}
\left(\frac{\partial}{\partial t}+2 \lambda \frac{\partial^{\frac{1}{2}}}{\partial t^{\frac{1}{2}}}\right) w_{\frac{1}{2}}^{1}(x, t)=c^{2} \frac{\partial^{2}}{\partial x^{2}} w_{\frac{1}{2}}^{1}(x, t), \quad x \in \mathbb{R}, t>0, \\
w_{\frac{1}{2}}^{1}(x, 0)=\delta(x),
\end{array}\right.
$$


has solution coinciding with the distribution of $T(|B(t)|), t>0$, where $|B(t)|$, $t>0$, is a reflecting Brownian motion independent from $T$ (see Orsingher and Beghin [13]). For $\lambda \rightarrow \infty, c \rightarrow \infty$, in such a way that $\frac{c^{2}}{\lambda} \rightarrow 1$ the fractional diffusion equation (1.1) is obtained from (1.21) and the composition $T(|B(t)|), t>$ 0 , converges in distribution to the iterated Brownian motion. Our result, specialized to this particular case gives the following unexpected equality in distribution

$$
T(|B(t)|) \stackrel{\text { law }}{=} B\left(c^{2} L^{\frac{1}{2}}(t)\right), \quad t>0
$$

where

$$
\operatorname{Pr}\left\{B\left(c^{2} \mathcal{L}^{\nu}(t)\right) \in d x\right\}=\frac{\lambda d x}{c \pi} \int_{0}^{t} \frac{1}{\sqrt{s(t-s)}} e^{-\frac{x^{2}}{4 c^{2} s}-\frac{\lambda^{2} s^{2}}{t-s}}\left(\frac{s}{2(t-s)}+1\right) d s,
$$

and

$$
\operatorname{Pr}\{T(|B(t)|) \in d x\}=\int_{0}^{\infty} \operatorname{Pr}\{T(s) \in d x\} \operatorname{Pr}\{|B(t)| \in d s\} .
$$

The absolutely continuous component of the distribution of the telegraph process $T(t), t>0$, reads

$$
\operatorname{Pr}\{T(s) \in d x\}=\frac{d x e^{-\lambda t}}{2 c}\left\{\lambda I_{0}\left(\frac{\lambda}{c} \sqrt{c^{2} t^{2}-x^{2}}\right)+\frac{\partial}{\partial t} I_{0}\left(\frac{\lambda}{c} \sqrt{c^{2} t^{2}-x^{2}}\right)\right\},
$$

where $|x|<c t, t>0, c>0$, and

$$
I_{0}(x)=\sum_{k=0}^{\infty}\left(\frac{x}{2}\right)^{2 k} \frac{1}{(k !)^{2}} .
$$

For $n=2, \beta=1$ and $\nu=1$, equation (1.4) coincides with that of damped planar vibrations (we call it planar telegraph equation) and governs the vertical oscillations of thin deformable structures. The solution to

$$
\left\{\begin{array}{l}
\left(\frac{\partial^{2}}{\partial t^{2}}+2 \lambda \frac{\partial}{\partial t}\right) r(x, y, t)=c^{2}\left(\frac{\partial^{2}}{\partial x^{2}}+\frac{\partial^{2}}{\partial y^{2}}\right) r(x, y, t), \quad x^{2}+y^{2}<c^{2} t^{2}, t>0 \\
r(x, y, 0)=\delta(x, y) \\
r_{t}(x, y, 0)=0
\end{array}\right.
$$

corresponds to the distribution $r(x, y, t)$ of the vector $\boldsymbol{T}(t)=(X(t), Y(t))$ related to a planar motion described in Orsingher and De Gregorio [15]. This random motion $\boldsymbol{T}(t), t>0$, is performed at finite velocity $c$, possesses sample paths composed by segments whose orientation is uniform in $(0,2 \pi)$, and with changes of direction at Poisson times. The distribution $r(x, y, t)$ of $\boldsymbol{T}(t), t>0$, is concentrated inside a circle $C_{c t}$ of radius $c t$ and has an absolutely continuous component which reads

$$
r(x, y, t)=\frac{\lambda}{2 \pi c} \frac{e^{-\lambda t+\frac{\lambda}{c} \sqrt{c^{2} t^{2}-\left(x^{2}+y^{2}\right)}}}{\sqrt{c^{2} t^{2}-\left(x^{2}+y^{2}\right)}}, \quad(x, y) \in C_{c t}, t>0 .
$$

If no Poisson event occurs, the moving particle reaches the boundary $\partial C_{c t}$ of $C_{c t}$ with probability $e^{-\lambda t}$. The vector process $\boldsymbol{T}(t), t>0$, taken at a random time represented by a reflecting Brownian motion, $|B(t)|$, has distribution

$$
q(x, y, t)=\int_{0}^{\infty} \operatorname{Pr}\{X(t) \in d s, Y(t) \in d s\} \operatorname{Pr}\{|B(t)| \in d s\}
$$


which satisfies the fractional equation

$$
\left(\frac{\partial}{\partial t}+2 \lambda \frac{\partial^{\frac{1}{2}}}{\partial t^{\frac{1}{2}}}\right) q(x, y, t)=c^{2}\left(\frac{\partial^{2}}{\partial x^{2}}+\frac{\partial^{2}}{\partial y^{2}}\right) q(x, y, t), \quad(x, y) \in \mathbb{R}^{2}, t>0 .
$$

However, the distribution of $\boldsymbol{B}_{2}\left(c^{2} \mathcal{L}^{\frac{1}{2}}(t)\right), t>0$, does not coincide with (1.29) ( $\boldsymbol{B}_{2}$ is a two dimensional Brownian motion). In this case the role of $T(t), t>0$, in (1.22) is here played by a process which is a slight modification of $\boldsymbol{T}(t), t>0$. We take the planar process with law

$\mathfrak{r}(x, y, t)=\frac{\lambda e^{-\lambda t}}{2 \pi c}\left[\frac{e^{\frac{\lambda}{c} \sqrt{c^{2} t^{2}-\left(x^{2}+y^{2}\right)}}+e^{-\frac{\lambda}{c} \sqrt{c^{2} t^{2}-\left(x^{2}+y^{2}\right)}}}{\sqrt{c^{2} t^{2}-\left(x^{2}+y^{2}\right)}}\right], \quad x^{2}+y^{2}<c^{2} t^{2}, t>0$,

which also solves equation (1.27). The process with distribution

$$
\begin{aligned}
\mathfrak{q}(x, y, t) & =\int_{0}^{\infty} \mathfrak{r}(x, y, s)\left[\operatorname{Pr}\{|B(t)| \in d s\}+\frac{1}{2 \lambda} \frac{\partial^{\frac{1}{2}}}{\partial t^{\frac{1}{2}}} \operatorname{Pr}\{|B(t)| \in d s\}\right] \\
& =\int_{0}^{\infty}\left(\mathfrak{r}(x, y, s)+\frac{\partial}{\partial s} \mathfrak{r}(x, y, s)\right) \operatorname{Pr}\{|B(t)| \in d s\}
\end{aligned}
$$

has the same law of a planar Brownian motion at the time $\mathcal{L}^{\frac{1}{2}}(t), t>0$. The process $\mathfrak{T}(t), t>0$, possessing distribution (1.31) is obtained from $\boldsymbol{T}(t), t>0$, by disregarding displacements started off by even-order Poisson events.

1.2. Notations. For the reader convenience we list below the main notations used throughout the paper.

- $S_{n}^{2 \beta}(t)=\left(S_{1}^{2 \beta}(t), S_{2}^{2 \beta}(t), \cdots, S_{n}^{2 \beta}(t)\right), t>0,0<\beta \leq 1, n \in \mathbb{N}$ is a isotropic stable $n$-dimensional process with law $v_{\beta}(\boldsymbol{x}, t), \boldsymbol{x} \in \mathbb{R}^{n}, t>0$.

- $H^{\nu}(t), t>0,0<\nu<1$, is a totally positively-skewed stable process (stable subordinator), with law $h_{\nu}(x, t), x \geq 0, t>0$.

- $L^{\nu}(t), t>0$, is the inverse of $H^{\nu}(t), t>0$, and has law $l_{\nu}(x, t), x \geq 0$, $t>0$.

- $\mathcal{H}^{\nu}(t)=H_{1}^{2 \nu}(t)+(2 \lambda)^{\frac{1}{\nu}} H_{2}^{\nu}(t), t>0$, is the sum of two independent stable subordinators and has law $h_{\nu}(x, t), x \geq 0, t>0$.

- $\mathcal{L}^{\nu}(t), t>0$, is the inverse of $\mathcal{H}^{\nu}(t), t>0$ and possesses distribution $\mathcal{l}_{\nu}(x, t)$, $x \geq 0, t>0$.

- $T(t), t>0$, is a telegraph process with parameters $c>0$ and $\lambda>0$ and law $p_{T}(x, t),-c t<x<c t, t>0$.

- $\boldsymbol{W}_{n}(t)=\boldsymbol{S}_{n}^{2 \beta}\left(c^{2} \mathcal{L}^{\nu}(t)\right), t>0$, has law $w_{\nu}^{\beta}(\boldsymbol{x}, t), \boldsymbol{x} \in \mathbb{R}^{n}, t>0$.

- $\mathcal{W}(t)=T(|B(t)|), t>0$, has distribution $w(x, t), x \in \mathbb{R}, t>0$.

- $\boldsymbol{T}(t), t>0$, is the planar process with infinite directions, parameters $c, \lambda>$ 0 and law $r(x, y, t),(x, y) \in C_{c t}=\left\{(x, y) \in \mathbb{R}^{2}: x^{2}+y^{2}<c^{2} t^{2}\right\}, t>0$.

- $\mathfrak{T}(t), t>0$, is the planar process with infinite directions, parameters $c, \lambda>$ 0 and law $\mathfrak{r}(x, y, t),(x, y) \in C_{c t}=\left\{(x, y) \in \mathbb{R}^{2}: x^{2}+y^{2}<c^{2} t^{2}\right\}, t>0$, constructed by disregading displacements started off only by even-labelled Poisson events.

- $\boldsymbol{Q}(t)=\boldsymbol{T}(|B(t)|), t>0$, has law $q(x, y, t),(x, y) \in \mathbb{R}^{2}, t>0$. 
- By $\widetilde{f}$ we denote the Laplace transform of the function $f$ and by $\widehat{f}$ we denote its Fourier transform.

1.3. Preliminaries. Let us consider a stable process $S^{\nu}(t), t>0,0<\nu \leq 2$, $\nu \neq 1$, with characteristic function

$$
\mathbb{E} e^{i \xi S^{\nu}(t)}=e^{-\sigma|\xi|^{\nu} t\left(1-i \theta \operatorname{sign}(\xi) \tan \frac{\nu \pi}{2}\right)}
$$

where $\theta \in[-1,1]$ is the skewness parameter and

$$
\sigma=\cos \frac{\pi \nu}{2}
$$

For $\theta=1$ the distribution corresponding to (1.33) is totally positively skewed and for $\theta=-1$ is totally negatively skewed. The stable process with stationary and independent increments, totally positively skewed will be denoted as $H^{\nu}(t), t>0$. We note that the density $h_{\nu}(x, t)$, of $H^{\nu}(t)$, is zero at $x=0$ as the following calculation show

$$
\begin{aligned}
h_{\nu}(0, t) & =\frac{1}{2 \pi} \int_{-\infty}^{\infty} \mathbb{E} e^{i \xi H^{\nu}(t)} d \xi=\frac{1}{2 \pi} \int_{-\infty}^{\infty} e^{-\sigma|\xi|^{\nu} t\left(1-i \tan \frac{\nu \pi}{2}\right)} d \xi \\
& =\frac{1}{2 \pi}\left[\int_{0}^{\infty} e^{-\sigma|\xi|^{\nu} t\left(1-i \tan \frac{\nu \pi}{2}\right)} d \xi+\int_{-\infty}^{0} e^{-\sigma|\xi|^{\nu} t\left(1+i \tan \frac{\nu \pi}{2}\right)} d \xi\right] \\
& =\frac{1}{2 \pi}\left[\int_{0}^{\infty} e^{-|\xi|^{\nu} t e^{-\frac{i \nu \pi}{2}}} d \xi+\int_{0}^{\infty} e^{-|\xi|^{\nu} t e^{\frac{i \nu \pi}{2}}} d \xi\right] \\
& =\frac{1}{2 \pi}\left[\int_{0}^{\infty} e^{-z}\left(\frac{z}{t}\right)^{\frac{1}{\nu}-1} e^{\frac{i \pi}{2}} d z+\int_{0}^{\infty} e^{-z}\left(\frac{z}{t}\right)^{\frac{1}{\nu}-1} \frac{1}{t} e^{-\frac{i \pi}{2}} d z\right] \\
& =\frac{\cos \frac{\pi}{2}}{\pi} \int_{0}^{\infty} e^{-z}\left(\frac{z}{t}\right)^{\frac{1}{\nu}-1} \frac{1}{t} d z=0 .
\end{aligned}
$$

The positively skewed stable r.v. $H^{\nu}(t)$ has $x$-Laplace transform

$$
\widetilde{h_{\nu}}(\mu, t)=\mathbb{E} e^{-\mu H^{\nu}(t)}=e^{-t \mu^{\nu}}, \quad 0<\nu<1,
$$

and therefore Fourier transform

$$
\begin{aligned}
\widehat{h_{\nu}}(\xi, t)=\mathbb{E} e^{i \xi H^{\nu}(t)} & =\mathbb{E}\left(e^{-(-i \xi) H^{\nu}(t)}\right)=e^{-t\left(|\xi| e^{-\frac{i \pi}{2} \operatorname{sign}(\xi)}\right)^{\nu}} \\
& =e^{-t|\xi|^{\nu} \cos \frac{\pi \nu}{2}\left(1-i \operatorname{sign}(\xi) \tan \frac{\pi \nu}{2}\right)} .
\end{aligned}
$$

This shows once again that the skeweness parameter is $\theta=1$.

The probability law $h_{\nu}(x, t)$, of $H^{\nu}(t), t>0$, solves the problem

$$
\left\{\begin{array}{l}
\left(\frac{\partial}{\partial t}+\frac{\partial^{\nu}}{\partial x^{\nu}}\right) h_{\nu}(x, t)=0, \quad x>0, t>0,0<\nu<1, \\
h_{\nu}(0, t)=0 \\
h_{\nu}(x, 0)=\delta(x) .
\end{array}\right.
$$

By taking the $x$-Laplace transform of the Riemann-Liouville fractional derivative appearing in (1.38) we have that

$$
\begin{aligned}
& \mathcal{L}\left[\frac{\partial^{\nu}}{\partial x^{\nu}} h_{\nu}(x, t)\right](\mu)=\int_{0}^{\infty} e^{-\mu x} \frac{\partial^{\nu}}{\partial x^{\nu}} h_{\nu}(x, t) d x \\
= & \int_{0}^{\infty} e^{-\mu x}\left[\frac{1}{\Gamma(1-\nu)} \frac{d}{d x} \int_{0}^{x} \frac{h_{\nu}(z, t)}{(x-z)^{\nu}} d z\right] d x
\end{aligned}
$$




$$
\begin{aligned}
& =\int_{0}^{\infty} e^{-\mu x}\left[\frac{1}{\Gamma(1-\nu)} \int_{0}^{x} \frac{d}{d x} \frac{h_{\nu}(x-z, t)}{z^{\nu}} d z+\frac{h_{\nu}(0, t)}{\Gamma(1-\nu) x^{\nu}}\right] d x \\
& =\frac{h_{\nu}(0, t)}{\Gamma(1-\nu)} \int_{0}^{\infty} e^{-\mu x} x^{1-\nu-1} d x+\frac{1}{\Gamma(1-\nu)} \int_{0}^{\infty} \frac{d z}{z^{\nu}} \int_{z}^{\infty} d x e^{-\mu x} \frac{d}{d x} h_{\nu}(x-z, t) \\
& =h_{\nu}(0, t) \mu^{\nu-1}+\frac{1}{\Gamma(1-\nu)} \int_{0}^{\infty} e^{-\mu z} z^{-\nu} d z \int_{0}^{\infty} e^{-\mu x} \frac{d}{d x} h_{\nu}(x, t) d x \\
& =h_{\nu}(0, t) \mu^{\nu-1}+\left[\int_{0}^{\infty} e^{-\mu x} h_{\nu}(x, t) d x\right] \mu \frac{1}{\mu^{1-\nu}}-\mu^{\nu-1} h_{\nu}(0, t)=\mu^{\nu} \widetilde{h_{\nu}}(\mu, t) .
\end{aligned}
$$

Therefore

$$
\left\{\begin{array}{l}
\frac{\partial}{\partial t} \widetilde{h_{\nu}}(\mu, t)+\mu^{\nu} \widetilde{h_{\nu}}(\mu, t)=0, \quad \mu>0, t>0, \\
\frac{h_{\nu}}{(\mu, 0)=1}
\end{array}\right.
$$

so that

$$
\widetilde{h_{\nu}}(\mu, t)=e^{-\mu^{\nu} t}
$$

In other words the density of a positively skewed stable r.v. solves the spacefractional problem (1.38).

We will also deal with the inverse process of $H^{\nu}(t), t>0$, say $L^{\nu}(t), t>0$, for which

$$
\operatorname{Pr}\left\{H^{\nu}(x)>t\right\}=\operatorname{Pr}\left\{L^{\nu}(t)<x\right\}, \quad x>0, t>0 .
$$

Such a process has non-negative, non-stationary and non-independent increments. Furthemore we recall that the law $l_{\nu}(x, t)$ of $L^{\nu}(t)$, can be written as

$$
l_{\nu}(x, t)=\frac{1}{t^{\nu}} W_{-\nu, 1-\nu}\left(-\frac{x}{t^{\nu}}\right), \quad x \geq 0, t>0
$$

where

$$
W_{a, b}(x)=\sum_{k=0}^{\infty} \frac{x^{k}}{k ! \Gamma(a k+b)}, \quad x \in \mathbb{R}, a>-1, b \in \mathbb{C},
$$

is the Wright function, and has Laplace transform

$$
\widetilde{l_{\nu}}(x, \mu)=\int_{0}^{\infty} e^{-\mu t} l_{\nu}(x, t) d t=\int_{0}^{\infty} e^{-\mu t} \frac{1}{t^{\nu}} W_{-\nu, 1-\nu}\left(-\frac{x}{t^{\nu}}\right) d t=\mu^{\nu-1} e^{-x \mu^{\nu}} .
$$

\section{Sum of Stable subordinators, $\mathscr{H}^{\nu}(t)=H_{1}^{2 \nu}(t)+(2 \lambda)^{\frac{1}{\nu}} H_{2}^{\nu}(t)$}

For the construction of the vector process $\boldsymbol{W}_{n}(t)=\boldsymbol{S}_{n}^{2 \beta}\left(c^{2} \mathcal{L}^{\nu}(t)\right), t>0$, whose distribution is driven by the general space-time fractional telegraph equation (1.4), we need the sum $\mathcal{H}^{\nu}(t), t>0$, of two independent positively skewed processes. The second step consists in constructing the process $\mathcal{L}^{\nu}(t), t>0$, inverse to $\mathcal{H}^{\nu}(t)$, $t>0$. We now start by considering the following sum

$$
\mathcal{H}^{\nu}(t)=H_{1}^{2 \nu}(t)+(2 \lambda)^{\frac{1}{\nu}} H_{2}^{\nu}(t), \quad t>0,0<\nu \leq \frac{1}{2},
$$

with $H_{1}^{2 \nu}, H_{2}^{\nu}$, independent, positively-skewed, stable random variables, $\lambda>0$. The distribution of $\mathcal{H}^{\nu}(t)$ can be written as

$$
\hbar_{\nu}(x, t)=\int_{0}^{x} h_{2 \nu}(y, t) h_{\nu}(x-y, 2 \lambda t) d y
$$


Taking the double Laplace transform of $(2.2)$, with respect to $t$ and $x$, we get

$$
\begin{aligned}
\widetilde{\widetilde{\kappa_{\nu}}}(\gamma, \mu) & =\int_{0}^{\infty} e^{-\mu t} \int_{0}^{\infty} e^{-\gamma x} h_{\nu}(x, t) d x d t=\int_{0}^{\infty} e^{-\mu t-t \gamma^{2 \nu}-2 \lambda t \gamma^{\nu}} d t \\
& =\frac{1}{\gamma^{2 \nu}+2 \lambda \gamma^{\nu}+\mu}=\left[\frac{1}{\gamma^{\nu}-r_{2}}-\frac{1}{\gamma^{\nu}-r_{1}}\right] \frac{1}{r_{2}-r_{1}}
\end{aligned}
$$

where, for $0<\mu<\lambda^{2}$,

$$
\left\{\begin{array}{l}
r_{1}=-\lambda-\sqrt{\lambda^{2}-\mu}, \\
r_{2}=-\lambda+\sqrt{\lambda^{2}-\mu} .
\end{array}\right.
$$

By means of formula

$$
\int_{0}^{\infty} e^{-\gamma x} x^{\alpha-1} E_{\alpha, \alpha}\left(\eta x^{\alpha}\right) d x=\frac{1}{\gamma^{\alpha}-\eta},
$$

where $E_{\nu, \nu}(z)$ is the Mittag-Leffler function defined in (1.9), we can invert the $x$-Laplace transform in (2.3) obtaining, for $\mu<\lambda^{2}$,

$$
\begin{aligned}
& \widetilde{\kappa}_{\nu}(x, \mu)= \\
= & \frac{x^{\nu-1}}{2 \sqrt{\lambda^{2}-\mu}}\left[E_{\nu, \nu}\left(\left(-\lambda+\sqrt{\lambda^{2}-\mu}\right) x^{\nu}\right)-E_{\nu, \nu}\left(\left(-\lambda-\sqrt{\lambda^{2}-\mu}\right) x^{\nu}\right)\right] \\
= & \frac{1}{2 \sqrt{\lambda^{2}-\mu}}\left[\frac{1}{-\lambda+\sqrt{\lambda^{2}-\mu}} \frac{\partial}{\partial x} E_{\nu, 1}\left(\left(-\lambda+\sqrt{\lambda^{2}-\mu}\right) x^{\nu}\right)\right. \\
& \left.-\frac{1}{-\lambda-\sqrt{\lambda^{2}-\mu}} \frac{\partial}{\partial x} E_{\nu, 1}\left(\left(-\lambda-\sqrt{\lambda^{2}-\mu}\right) x^{\nu}\right)\right] .
\end{aligned}
$$

Formula (2.6) gives the explicit form of the $t$-Laplace transform of $\hbar_{\nu}(x, t)$ in terms of Mittag-Leffler functions. In view of formula

$$
E_{\nu, 1}\left(-\lambda t^{\nu}\right)=\frac{1}{\pi} \int_{0}^{\infty} \frac{e^{-\lambda^{\frac{1}{\nu}} t x} x^{\nu-1} \sin \pi \nu}{x^{2 \nu}+1+2 x^{\nu} \cos \pi \nu} d x, \quad 0<\nu<1,
$$

we have that

$$
\begin{aligned}
& \widetilde{\kappa}_{\nu}(x, \mu)= \frac{1}{2 \sqrt{\lambda^{2}-\mu}}\left[\frac{1}{-\lambda+\sqrt{\lambda^{2}-\mu}} \frac{\partial}{\partial x} \int_{0}^{\infty} \frac{e^{-x y\left(\lambda-\sqrt{\lambda^{2}-\mu}\right)^{\frac{1}{\nu}}} y^{\nu-1} \sin \pi \nu d y}{\pi\left(y^{2 \nu}+1+2 y^{\nu} \cos \pi \nu\right)}\right. \\
&\left.+\frac{1}{\lambda+\sqrt{\lambda^{2}-\mu}} \frac{\partial}{\partial x} \int_{0}^{\infty} \frac{e^{-x y\left(\lambda+\sqrt{\lambda^{2}-\mu}\right)^{\frac{1}{\nu}}} y^{\nu-1} \sin \pi \nu d y}{\pi\left(y^{2 \nu}+1+2 y^{\nu} \cos \pi \nu\right)}\right] \\
&= \int_{0}^{\infty} \frac{d y y^{\nu} \sin \pi \nu}{\pi\left(y^{2 \nu}+1+2 y^{\nu} \cos \pi \nu\right)} \frac{1}{2 \sqrt{\lambda^{2}-\mu}}\left[\left(\lambda-\sqrt{\lambda^{2}-\mu}\right)^{\frac{1}{\nu}-1} .\right. \\
& \cdot e^{-x y\left(\lambda-\sqrt{\lambda^{2}-\mu}\right)^{\frac{1}{\nu}}-\left(\lambda+\sqrt{\lambda^{2}-\mu}\right)^{\frac{1}{\nu}-1} e^{\left.-x y\left(\lambda+\sqrt{\lambda^{2}-\mu}\right)^{\frac{1}{\nu}}\right]}} \\
&=\mathbb{E}\left\{\frac{u^{\nu}}{2 \sqrt{\lambda^{2}-\mu}}\left[\left(-r_{2}\right)^{\frac{1}{\nu}-1} e^{-x \mathcal{U}^{\nu}\left(-r_{2}\right)^{\frac{1}{\nu}}}-\left(-r_{1}\right)^{\frac{1}{\nu}-1} e^{-x \mathcal{u}^{\nu}\left(-r_{1}\right)^{\frac{1}{\nu}}}\right]\right\}
\end{aligned}
$$




$$
=\frac{1}{r_{2}-r_{1}} \frac{\partial}{\partial x} \mathbb{E}\left[\frac{e^{-x u^{\nu}\left(-r_{2}\right)^{\frac{1}{\nu}}}}{r_{2}}-\frac{e^{-x \mathcal{U}^{\nu}\left(-r_{1}\right)^{\frac{1}{\nu}}}}{r_{1}}\right],
$$

where $\mathcal{U}^{\nu}$ is the Lamperti distribution with density

$$
\frac{\operatorname{Pr}\left\{u^{\nu} \in d u\right\}}{d u}=\frac{\sin \pi \nu}{\pi} \frac{u^{\nu-1}}{1+u^{2 \nu}+2 u^{\nu} \cos \pi \nu}, \quad u>0,
$$

and represents the law of the ratio of two independent stable r.v.'s of the same order $\nu$.

Theorem 2.1. The law $f_{\nu}(x, t)$ of the process $\mathcal{H}^{\nu}(t)=H_{1}^{2 \nu}(t)+(2 \lambda)^{\frac{1}{\nu}} H_{2}^{\nu}(t)$ solves the fractional problem

$$
\left\{\begin{array}{l}
\frac{\partial}{\partial t} h_{\nu}(x, t)=-\left(\frac{\partial^{2 \nu}}{\partial x^{2 \nu}}+2 \lambda \frac{\partial^{\nu}}{\partial x^{\nu}}\right) h_{\nu}(x, t), \quad x>0, t>0,0<\nu<\frac{1}{2}, \\
h_{\nu}(0, t)=0 \\
h_{\nu}(x, 0)=\delta(x) .
\end{array}\right.
$$

The fractional derivatives appearing in (2.10) are intended in the Riemann-Liouville sense.

Proof. By considering (1.37), we have that the Fourier transform of $\hbar_{\nu}(x, t)$ is written as

$$
\begin{aligned}
\widehat{\hat{h}_{\nu}}(\xi, t) & =\mathbb{E} e^{i \xi \mathcal{H}^{\nu}(t)}=\mathbb{E} e^{i \xi\left[H^{2 \nu}(t)+(2 \lambda)^{\frac{1}{\nu}} H^{\nu}(t)\right]}=\mathbb{E} e^{i \xi H^{2 \nu}(t)} e^{i \xi H^{\nu}(2 \lambda t)} \\
& =e^{-t|\xi|^{2 \nu} \cos \pi \nu(1-i \operatorname{sign}(\xi) \tan \pi \nu)-2 \lambda t|\xi|^{\nu} \cos \frac{\pi \nu}{2}\left(1-i \operatorname{sign}(\xi) \tan \frac{\pi \nu}{2}\right)} \\
& =e^{-t\left(|\xi| e^{-\frac{i \pi}{2} \operatorname{sign}(\xi)}\right)^{2 \nu}-2 \lambda t\left(|\xi| e^{-\frac{i \pi}{2} \operatorname{sign}(\xi)}\right)^{\nu}},
\end{aligned}
$$

and thus

$$
\begin{aligned}
\frac{\partial}{\partial t} \widehat{h}_{\nu}(\xi, t)= & {\left[-\left(|\xi| e^{-\frac{i \pi}{2} \operatorname{sign}(\xi)}\right)^{2 \nu}-2 \lambda\left(|\xi| e^{-\frac{i \pi}{2} \operatorname{sign}(\xi)}\right)^{\nu}\right] . } \\
& \cdot e^{-t\left(|\xi| e^{-\frac{i \pi}{2} \operatorname{sign}(\xi)}\right)^{2 \nu}-2 \lambda t\left(|\xi| e^{-\frac{i \pi}{2} \operatorname{sign}(\xi)}\right)^{\nu}}
\end{aligned}
$$

In view of the relationship

$$
|\xi| e^{-\frac{i \pi}{2} \operatorname{sign}(\xi)}=-i \xi
$$

we have that formula (2.12) can be rewritten as

$$
\frac{\partial}{\partial t} \widehat{h_{\nu}}(\xi, t)=\left[-(-i \xi)^{2 \nu}-2 \lambda(-i \xi)^{\nu}\right] e^{-t(-i \xi)^{2 \nu}-2 \lambda t(-i \xi)^{\nu}} .
$$

In (1.39) we have shown that

$$
\mathcal{L}\left[\frac{\partial^{\nu}}{\partial x^{\nu}} h_{\nu}(x, t)\right](\mu)=\int_{0}^{\infty} e^{-\mu x} \frac{\partial^{\nu}}{\partial x^{\nu}} h_{\nu}(x, t) d x=\mu^{\nu} \widetilde{h_{\nu}}(\mu, t)
$$

and thus for a sufficiently good function $f$ we have the following Fourier transform

$$
\mathcal{F}\left[\frac{\partial^{\nu}}{\partial x^{\nu}} f(x)\right](\xi)=\int_{0}^{\infty} e^{-(-i \xi) x} \frac{\partial^{\nu}}{\partial x^{\nu}} f(x) d x=(-i \xi)^{\nu} \widehat{f}(\xi) .
$$

In view of (2.16) we have that the Fourier transform of the right-hand side of the equation (2.10), equipped with the boundary conditions, is written as

$$
-\mathcal{F}\left[\frac{\partial^{2 \nu}}{\partial x^{2 \nu}} h_{\nu}(x, t)+2 \lambda \frac{\partial^{\nu}}{\partial x^{\nu}} h_{\nu}(x, t)\right](\xi)=
$$




$$
\begin{aligned}
& =-\int_{0}^{\infty} e^{-(-i \xi) x} \frac{\partial^{2 \nu}}{\partial x^{2 \nu}} \kappa_{\nu}(x, t) d x-2 \lambda \int_{0}^{\infty} e^{-(-i \xi) x} \frac{\partial^{\nu}}{\partial x^{\nu}} \hbar_{\nu}(x, t) d x \\
& =-\left((-i \xi)^{2 \nu}+2 \lambda(-i \xi)^{\nu}\right) \widehat{\epsilon}_{\nu}(\xi, t) \\
& =-\left((-i \xi)^{2 \nu}+2 \lambda(-i \xi)^{\nu}\right) e^{-t\left(|\xi| e^{-\frac{i \pi}{2} \operatorname{sign}(\xi)}\right)^{2 \nu}-2 \lambda t\left(|\xi| e^{-\frac{i \pi}{2} \operatorname{sign}(\xi)}\right)^{\nu}} \\
& =-\left((-i \xi)^{2 \nu}+2 \lambda(-i \xi)^{\nu}\right) e^{-t(-i \xi)^{2 \nu}-2 \lambda t(-i \xi)^{\nu}}
\end{aligned}
$$

which coincides with formula (2.14). This is tantamount to saying that the Fourier transform $\widehat{h_{\nu}}(\xi, t)$ is the solution to

$$
\left\{\begin{array}{l}
\frac{\partial}{\partial t} \widehat{h}_{\nu}(\xi, t)=-\left((-i \xi)^{2 \nu}+2 \lambda(-i \xi)^{\nu}\right) \widehat{h}_{\nu}(\xi, t), \quad \xi \in \mathbb{R}, t>0, \\
\widehat{h_{\nu}}(\xi, 0)=1
\end{array}\right.
$$

and this completes the proof.

2.1. The inverse process $\mathcal{L}^{\nu}(t)$. Let $\mathcal{L}^{\nu}(t), t>0$, be the inverse process of $\mathcal{H}^{\nu}(t)$, $t>0$, as defined in (1.13) for which

$$
\operatorname{Pr}\left\{\mathcal{L}^{\nu}(t)<x\right\}=\operatorname{Pr}\left\{\mathcal{H}^{\nu}(x)>t\right\}, \quad x, t>0,
$$

and let $\mathcal{L}_{\nu}(x, t)$ be the law of $\mathcal{L}^{\nu}(t), t>0$. We have the following result.

Theorem 2.2. The law $\mathcal{L}_{\nu}(x, t)$ of the process $\mathcal{L}^{\nu}(t), t>0$, solves the timefractional boundary-initial problem

$$
\left\{\begin{array}{l}
\left(\frac{\partial^{2 \nu}}{\partial t^{2 \nu}}+2 \lambda \frac{\partial^{\nu}}{\partial t^{\nu}}\right) \zeta_{\nu}(x, t)=-\frac{\partial}{\partial x} \varsigma_{\nu}(x, t), \quad x>0, t>0,0<\nu<\frac{1}{2}, \\
\iota_{\nu}(x, 0)=\delta(x), \\
\iota_{\nu}(0, t)=\frac{t^{-2 \nu}}{\Gamma(1-2 \nu)}+2 \lambda \frac{t^{-\nu}}{\Gamma(1-\nu)},
\end{array}\right.
$$

and has $x$-Laplace transform which reads, for $0<\gamma<\lambda^{2}$,

$$
\tilde{\varsigma}_{\nu}(\gamma, t)=\frac{1}{2}\left[\left(1+\frac{\lambda}{\sqrt{\lambda^{2}-\gamma}}\right) E_{\nu, 1}\left(r_{1} t^{\nu}\right)+\left(1-\frac{\lambda}{\sqrt{\lambda^{2}-\gamma}}\right) E_{\nu, 1}\left(r_{2} t^{\nu}\right)\right],
$$

where

$$
r_{1}=-\lambda+\sqrt{\lambda^{2}-\gamma}, \quad r_{2}=-\lambda-\sqrt{\lambda^{2}-\gamma}
$$

The fractional derivatives appearing in (2.20) are intended in the Riemann-Liouville sense.

Proof. We first show that the analytical solution to the problem (2.20) has double Laplace transform $\widetilde{\widetilde{\varphi}}_{\nu}(\gamma, \mu)$ written as

$$
\widetilde{\widetilde{c}}_{\nu}(\gamma, \mu)=\frac{\mu^{2 \nu-1}+2 \lambda \mu^{\nu-1}}{\mu^{2 \nu}+2 \lambda \mu^{\nu}+\gamma} .
$$

By taking the $t$-Laplace transform of the equation in (2.20) we have that

$$
\mu^{2 \nu}{\widetilde{\epsilon_{\nu}}}_{\nu}(x, \mu)+2 \lambda \mu^{\nu}{\widetilde{\iota_{\nu}}}_{\nu}(x, \mu)=-\frac{\partial}{\partial x} \widetilde{\boldsymbol{c}}_{\nu}(x, \mu) .
$$

By taking into account the boundary condition and performing the $x$-Laplace transform of (2.24) we have that

$$
\left(\mu^{2 \nu}+2 \lambda \mu^{\nu}\right) \widetilde{\widetilde{L}}_{\nu}(\gamma, \mu)=\widetilde{\zeta}_{\nu}(0, \mu)-\gamma \widetilde{\widetilde{L}}_{\nu}(\gamma, \mu)
$$


Now, by considering the boundary condition, we get that

$$
\begin{aligned}
\tilde{\iota}_{\nu}(0, \mu) & =\int_{0}^{\infty} d t e^{-\mu t} \zeta_{\nu}(0, t)=\int_{0}^{\infty} d t e^{-\mu t}\left[\frac{t^{-2 \nu}}{\Gamma(1-2 \nu)}+2 \lambda \frac{t^{-\nu}}{\Gamma(1-\nu)}\right] \\
& =\mu^{2 \nu-1}+2 \lambda \mu^{\nu-1}
\end{aligned}
$$

and thus

$$
\widetilde{\widetilde{L}}_{\nu}(\gamma, \mu)=\frac{\mu^{2 \nu-1}+2 \lambda \mu^{\nu-1}}{\mu^{2 \nu}+2 \lambda \mu^{\nu}+\gamma} .
$$

Now we show that the double Laplace transform of the law $\varsigma_{\nu}(x, t)$ coincides with (2.23). We first recall that

$$
\begin{aligned}
\widetilde{h_{\nu}}(\mu, x) & =\int_{0}^{\infty} d t e^{-\mu t} h_{\nu}(t, x)=\mathbb{E} e^{-\mu \mathcal{H}^{\nu}(x)}=\mathbb{E} e^{-\mu H^{2 \nu}(x)} \mathbb{E} e^{-\mu H^{\nu}(2 \lambda x)} \\
& =\widetilde{h_{2 \nu}}(\mu, x) \widetilde{h_{\nu}}(\mu, 2 \lambda x)=e^{-x \mu^{2 \nu}-x 2 \lambda \mu^{\nu}}, \quad x>0,
\end{aligned}
$$

where we used result (1.36). By considering the construction of the process $\mathcal{L}^{\nu}(t)$, $t>0$, as the inverse process of $\mathcal{H}^{\nu}(t), t>0$, as stated in (2.19), we get

$$
\zeta_{\nu}(x, t)=\frac{\operatorname{Pr}\left\{\mathcal{L}^{\nu}(t) \in d x\right\}}{d x}=-\frac{\partial}{\partial x} \operatorname{Pr}\left\{\mathcal{H}^{\nu}(x)<t\right\}=-\frac{\partial}{\partial x} \int_{0}^{t} \hbar_{\nu}(s, x) d s .
$$

In view of (2.29), the double Laplace transform of $\mathcal{L}_{\nu}(x, t)$ can be obtained observing that

$$
\begin{aligned}
\widetilde{\widetilde{c}}_{\nu}(\gamma, \mu) & =\int_{0}^{\infty} d x e^{-\gamma x} \int_{0}^{\infty} d t e^{-\mu t}\left[-\frac{\partial}{\partial x} \int_{0}^{t} \hbar_{\nu}(s, x) d s\right] \\
& =-\int_{0}^{\infty} d x e^{-\gamma x} \frac{\partial}{\partial x} \int_{0}^{\infty} d t e^{-\mu t} \int_{0}^{t} \hbar_{\nu}(s, x) d s \\
& =-\frac{1}{\mu} \int_{0}^{\infty} d x e^{-\gamma x} \frac{\partial}{\partial x} \widetilde{h}_{\nu}(x, \mu)=-\frac{1}{\mu} \int_{0}^{\infty} d x e^{-\gamma x}\left[\frac{\partial}{\partial x} e^{-x \mu^{2 \nu}-2 \lambda x \mu^{\nu}}\right] \\
& =\left(\mu^{2 \nu-1}+2 \lambda \mu^{\nu-1}\right) \int_{0}^{\infty} d x e^{-\gamma x-x \mu^{2 \nu}-2 \lambda x \mu^{\nu}}=\frac{\mu^{2 \nu-1}+2 \lambda \mu^{\nu-1}}{\mu^{2 \nu}+2 \lambda \mu^{\nu}+\gamma}
\end{aligned}
$$

which coincides with (2.23). Now we pass to the derivation of the $x$-Laplace transform of $l_{\nu}(x, t)$. We can write

$$
\begin{aligned}
\widetilde{\widetilde{L}}_{\nu}(\gamma, \mu) & =\frac{\mu^{2 \nu-1}+2 \lambda \mu^{\nu-1}}{\mu^{2 \nu}+2 \lambda \mu^{\nu}+\gamma}=\frac{\mu^{\nu-1}}{\mu^{\nu}-r_{1}}+\frac{\mu^{\nu-1}}{\mu^{\nu}-r_{2}}-\frac{\mu^{2 \nu-1}}{\left(\mu^{\nu}-r_{1}\right)\left(\mu^{\nu}-r_{2}\right)} \\
& =\frac{\mu^{\nu-1}}{\mu^{\nu}-r_{1}}+\frac{\mu^{\nu-1}}{\mu^{\nu}-r_{2}}-\left[\frac{\mu^{\nu-(1-\nu)}}{\mu^{\nu}-r_{1}}-\frac{\mu^{\nu-(1-\nu)}}{\mu^{\nu}-r_{2}}\right] \frac{1}{2 \sqrt{\lambda^{2}-\gamma}},
\end{aligned}
$$

where

$$
r_{1}=-\lambda+\sqrt{\lambda^{2}-\gamma}, \quad r_{2}=-\lambda-\sqrt{\lambda^{2}-\gamma}
$$

Now we need the following results

$$
\begin{aligned}
& \int_{0}^{\infty} e^{-\mu t} E_{\nu, 1}\left(r_{j} t^{\nu}\right) d t=\frac{\mu^{\nu-1}}{\mu^{\nu}-r_{j}}, \quad j=1,2, \\
& \int_{0}^{\infty} e^{-\mu t} t^{(1-\nu)-1} E_{\nu, 1-\nu}\left(r_{j} t^{\nu}\right) d t=\frac{\mu^{2 \nu-1}}{\mu^{\nu}-r_{j}} .
\end{aligned}
$$


Therefore

$$
\widetilde{\zeta}_{\nu}(\gamma, t)=E_{\nu, 1}\left(r_{1} t^{\nu}\right)+E_{\nu, 1}\left(r_{2} t^{\nu}\right)-\frac{t^{-\nu}}{2 \sqrt{\lambda^{2}-\gamma}}\left[E_{\nu, 1-\nu}\left(r_{1} t^{\nu}\right)-E_{\nu, 1-\nu}\left(r_{2} t^{\nu}\right)\right] .
$$

Since

$$
E_{\nu, 1-\nu}(z)=z E_{\nu, 1}(z)+\frac{1}{\Gamma(1-\nu)}
$$

we have that

$$
\begin{aligned}
\tilde{\iota}_{\nu}(\gamma, t) & =E_{\nu, 1}\left(r_{1} t^{\nu}\right)+E_{\nu, 1}\left(r_{2} t^{\nu}\right)-\frac{t^{-\nu}}{2 \sqrt{\lambda^{2}-\gamma}}\left[r_{1} t^{\nu} E_{\nu, 1}\left(r_{1} t^{\nu}\right)-r_{2} t^{\nu} E_{\nu, 1}\left(r_{2} t^{\nu}\right)\right] \\
& =\left(1-\frac{-\lambda+\sqrt{\lambda^{2}-\gamma}}{2 \sqrt{\lambda^{2}-\gamma}}\right) E_{\nu, 1}\left(r_{1} t^{\nu}\right)+\left(1-\frac{\lambda+\sqrt{\lambda^{2}-\gamma}}{2 \sqrt{\lambda^{2}-\gamma}}\right) E_{\nu, 1}\left(r_{2} t^{\nu}\right) \\
& =\frac{1}{2}\left[\left(1+\frac{\lambda}{\sqrt{\lambda^{2}-\gamma}}\right) E_{\nu, 1}\left(r_{1} t^{\nu}\right)+\left(1-\frac{\lambda}{\sqrt{\lambda^{2}-\gamma}}\right) E_{\nu, 1}\left(r_{2} t^{\nu}\right)\right],
\end{aligned}
$$

which coincides with (2.21).

Now we check that the Laplace transform (2.36) solves the fractional equation

$$
\begin{aligned}
\left(\frac{\partial^{2 \nu}}{\partial t^{2 \nu}}+2 \lambda \frac{\partial^{\nu}}{\partial t^{\nu}}\right) \tilde{\varphi}_{\nu}(\gamma, t) & =-\gamma \widetilde{L}_{\nu}(\gamma, t)+\varsigma_{\nu}(0, t) \\
& =-\gamma{\widetilde{\iota_{\nu}}}_{\nu}(\gamma, t)+\frac{t^{-2 \nu}}{\Gamma(1-2 \nu)}+2 \lambda \frac{t^{-\nu}}{\Gamma(1-\nu)}
\end{aligned}
$$

which is the $x$-Laplace transform of the equation appearing in (2.20). Since

$$
\begin{gathered}
\frac{\partial^{2 \nu}}{\partial t^{2 \nu}} \widetilde{L}_{\nu}(\gamma, t)-\frac{t^{-2 \nu}}{\Gamma(1-2 \nu)}=\frac{{ }^{C} \partial^{2 \nu}}{\partial t^{2 \nu}} \widetilde{L}_{\nu}(\gamma, t) \\
\frac{\partial^{\nu}}{\partial t^{\nu}} \widetilde{L}_{\nu}(\gamma, t)-\frac{t^{-\nu}}{\Gamma(1-\nu)}=\frac{{ }^{2} \partial^{\nu}}{\partial t^{\nu}} \widetilde{L}_{\nu}(\gamma, t)
\end{gathered}
$$

we therefore need to show that

$$
\left(\frac{{ }^{C} \partial^{2 \nu}}{\partial t^{2 \nu}}+2 \lambda \frac{{ }^{C} \partial^{\nu}}{\partial t^{\nu}}\right) \tilde{l}_{\nu}(\gamma, t)=-\gamma \widetilde{\tau_{\nu}}(\gamma, t) .
$$

In light of

$$
\begin{aligned}
& \frac{{ }^{C} \partial^{\nu}}{\partial t^{\nu}} E_{\nu, 1}\left(r_{j} t^{\nu}\right)=r_{j} E_{\nu, 1}\left(r_{j} t^{\nu}\right), \quad j=1,2, \\
& \frac{{ }^{C} \partial^{2 \nu}}{\partial t^{2 \nu}} E_{\nu, 1}\left(r_{j} t^{\nu}\right)=r_{j}^{2} E_{\nu, 1}\left(r_{j} t^{\nu}\right)+\frac{t^{-\nu} r_{j}}{\Gamma(1-\nu)},
\end{aligned}
$$

we are able to show that (2.21) solves (2.37). We first check result (2.42) as follows, for $0<2 \nu<1$

$$
\begin{aligned}
& \frac{{ }^{C} \partial^{2 \nu}}{\partial t^{2 \nu}} E_{\nu, 1}\left(r_{j} t^{\nu}\right)=\sum_{k=0}^{\infty} \frac{r_{j}^{k}}{\Gamma(\nu k+1)} \frac{{ }^{C} \partial^{2 \nu}}{\partial t^{2 \nu}} t^{\nu k} \\
= & \sum_{k=1}^{\infty} \frac{r_{j}^{k}}{\Gamma(\nu k+1)} \frac{\nu k}{\Gamma(1-2 \nu)} \int_{0}^{t} s^{\nu k-1}(t-s)^{-2 \nu} d s
\end{aligned}
$$




$$
\begin{aligned}
& =\sum_{k=1}^{\infty} \frac{r_{j}^{k} t^{\nu k-2 \nu}}{\Gamma(\nu k)} \frac{1}{\Gamma(1-2 \nu)} \int_{0}^{1} s^{\nu k-1}(1-s)^{1-2 \nu-1} d s \\
& =\sum_{k=1}^{\infty} \frac{r_{j}^{k} t^{\nu k-2 \nu}}{\Gamma(\nu k-2 \nu+1)}=\sum_{k=0}^{\infty} \frac{r_{j}^{k+1} t^{\nu k-\nu}}{\Gamma(\nu k-\nu+1)} \\
& =r_{j} t^{-\nu}\left[\sum_{k=1}^{\infty} \frac{\left(r_{j} t^{\nu}\right)^{k}}{\Gamma(\nu k-\nu+1)}+\frac{1}{\Gamma(1-\nu)}\right]=r_{j}^{2} E_{\nu, 1}\left(r_{j} t^{\nu}\right)+\frac{t^{-\nu} r_{j}}{\Gamma(1-\nu)} .
\end{aligned}
$$

Therefore

$$
\begin{aligned}
& \left(\frac{{ }^{C} \partial^{2 \nu}}{\partial t^{2 \nu}}+2 \lambda \frac{{ }^{C} \partial^{\nu}}{\partial t^{\nu}}\right) \widetilde{L}_{\nu}(\gamma, t)= \\
& =\frac{1}{2}\left[\left(1+\frac{\lambda}{\sqrt{\lambda^{2}-\gamma}}\right) \frac{{ }^{C} \partial^{2 \nu}}{\partial t^{2 \nu}} E_{\nu, 1}\left(r_{1} t^{\nu}\right)+\left(1-\frac{\lambda}{\sqrt{\lambda^{2}-\gamma}}\right) \frac{{ }^{C} \partial^{2 \nu}}{\partial t^{2 \nu}} E_{\nu, 1}\left(r_{2} t^{\nu}\right)\right] \\
& +2 \lambda \frac{1}{2}\left[\left(1+\frac{\lambda}{\sqrt{\lambda^{2}-\gamma}}\right) \frac{{ }^{C} \partial^{\nu}}{\partial t^{\nu}} E_{\nu, 1}\left(r_{1} t^{\nu}\right)+\left(1-\frac{\lambda}{\sqrt{\lambda^{2}-\gamma}}\right) \frac{{ }^{C} \partial^{\nu}}{\partial t^{\nu}} E_{\nu, 1}\left(r_{2} t^{\nu}\right)\right] \\
& =\frac{1}{2}\left[\left(1+\frac{\lambda}{\sqrt{\lambda^{2}-\gamma}}\right)\left(r_{1}^{2} E_{\nu, 1}\left(r_{1} t^{\nu}\right)+\frac{t^{-\nu} r_{1}}{\Gamma(1-\nu)}\right)\right. \\
& \left.+\left(1-\frac{\lambda}{\sqrt{\lambda^{2}-\gamma}}\right)\left(r_{2}^{2} E_{\nu, 1}\left(r_{2} t^{\nu}\right)+\frac{t^{-\nu} r_{2}}{\Gamma(1-\nu)}\right)\right] \\
& +2 \lambda \frac{1}{2}\left[\left(1+\frac{\lambda}{\sqrt{\lambda^{2}-\gamma}}\right)\left(r_{1} E_{\nu, 1}\left(r_{1} t^{\nu}\right)\right)+\left(1-\frac{\lambda}{\sqrt{\lambda^{2}-\gamma}}\right) r_{2} E_{\nu, 1}\left(r_{2} t^{\nu}\right)\right] \\
& =\frac{1}{2}\left[r_{1}\left(1+\frac{\lambda}{\sqrt{\lambda^{2}-\gamma}}\right) E_{\nu, 1}\left(r_{1} t^{\nu}\right)\left(r_{1}+2 \lambda\right)+r_{2}\left(1-\frac{\lambda}{\sqrt{\lambda^{2}-\gamma}}\right)\right. \text {. } \\
& \text { - } \left.E_{\nu, 1}\left(r_{2} t^{\nu}\right)\left(r_{2}+2 \lambda\right)\right] \\
& =-\frac{\gamma}{2} \frac{\lambda+\sqrt{\lambda^{2}-\gamma}}{\sqrt{\lambda^{2}-\gamma}} E_{\nu, 1}\left(r_{1} t^{\nu}\right)-\frac{\gamma}{2} \frac{\sqrt{\lambda^{2}-\gamma}-\lambda}{\sqrt{\lambda^{2}-\gamma}} E_{\nu, 1}\left(r_{2} t^{\nu}\right) \\
& =-\gamma\left[\frac{1}{2}\left[\left(1+\frac{\lambda}{\sqrt{\lambda^{2}-\gamma}}\right) E_{\nu, 1}\left(r_{1} t^{\nu}\right)+\left(1-\frac{\lambda}{\sqrt{\lambda^{2}-\gamma}}\right) E_{\nu, 1}\left(r_{2} t^{\nu}\right)\right]\right] \\
& =-\gamma \widetilde{l}_{\nu}(\gamma, t) \text {. }
\end{aligned}
$$

In the last steps we used the fact that

$$
\left(1+\frac{\lambda}{\sqrt{\lambda^{2}-\gamma}}\right) \frac{r_{1} t^{-\nu}}{\Gamma(1-\nu)}+\left(1-\frac{\lambda}{\sqrt{\lambda^{2}-\gamma}}\right) \frac{r_{2} t^{-\nu}}{\Gamma(1-\nu)}=0
$$

and

$$
r_{1}+2 \lambda=-r_{2}, \quad r_{2}+2 \lambda=-r_{1}, \quad r_{1} r_{2}=\gamma
$$


Remark 2.1. The derivation of result (2.21) suggests an alternative proof for the Fourier transform (Theorem 2.2 in Orsingher and Beghin [13]) of the law of the time-fractional telegraph process.

Remark 2.2. From (2.31) we get the time Laplace transform of $\varsigma_{\nu}(x, t)$, for $x>$ $0, \mu>0,0<\nu<\frac{1}{2}$, as

$$
\tilde{G}_{\nu}(x, \mu)=\mu^{2 \nu-1} e^{-x \mu^{2 \nu}} e^{-2 \lambda x \mu^{\nu}}+2 \lambda \mu^{\nu-1} e^{-2 \lambda x \mu^{\nu}} e^{-x \mu^{2 \nu}} .
$$

Since (see formulas (1.43) and (1.45))

$$
\widetilde{l_{\nu}}(x, \mu)=\int_{0}^{\infty} e^{-\mu t} \frac{1}{t^{\nu}} W_{-\nu, 1-\nu}\left(-\frac{x}{t^{\nu}}\right) d t=\mu^{\nu-1} e^{-x \mu^{\nu}}
$$

and (see formula (1.41))

$$
\widetilde{h_{\nu}}(\mu, t)=\int_{0}^{\infty} e^{-\mu x} h_{\nu}(x, t) d x=e^{-t \mu^{\nu}},
$$

we are able to invert (2.47) and we obtain the explicit distribution of the process $\mathcal{L}^{\nu}(t), t>0$, which reads

$$
\begin{aligned}
\mathcal{L}_{\nu}(x, t)= & \frac{\operatorname{Pr}\left\{\mathcal{L}^{\nu}(t) \in d x\right\}}{d x} \\
= & \int_{0}^{t} l_{2 \nu}(x, s) h_{\nu}(t-s, 2 \lambda x) d s+2 \lambda \int_{0}^{t} l_{\nu}(2 \lambda x, s) h_{2 \nu}(t-s, x) d s \\
= & \int_{0}^{t} \frac{1}{s^{2 \nu}} W_{-2 \nu, 1-2 \nu}\left(-\frac{x}{s^{2 \nu}}\right) h_{\nu}(t-s, 2 \lambda x) d s \\
& +2 \lambda \int_{0}^{t} \frac{1}{s^{\nu}} W_{-\nu, 1-\nu}\left(-\frac{2 \lambda x}{s^{\nu}}\right) h_{2 \nu}(t-s, x) d s .
\end{aligned}
$$

The densities $h_{\nu}$ and $h_{2 \nu}$ can be written down in terms of series expansion of stable laws (see pag. 245 of Orsingher and Beghin [16]).

\section{3. $n$-DIMENSIONAL STABLE LAWS AND FRACTIONAL LAPLACIAN}

Let

$$
S_{n}^{2 \beta}(t)=\left(S_{1}^{2 \beta}(t), S_{2}^{2 \beta}(t), \cdots, S_{n}^{2 \beta}(t)\right), \quad t>0, \beta \in(0,1],
$$

be the isotropic stable $n$-dimensional process with joint characteristic function

$$
\begin{aligned}
\widehat{v_{n}^{2 \beta}}(\boldsymbol{\xi}, t) & =\widehat{v_{n}^{2 \beta}}\left(\xi_{1}, \xi_{2}, \cdots, \xi_{n}, t\right)=\mathbb{E} e^{i \boldsymbol{\xi} \cdot \boldsymbol{S}_{n}^{2 \beta}(t)}=e^{-t\left(\sqrt{\xi_{1}^{2}+\xi_{2}^{2}+\cdots+\xi_{n}^{2}}\right)^{2 \beta}} \\
& =e^{-t\|\boldsymbol{\xi}\|^{2 \beta}} .
\end{aligned}
$$

The density corresponding to the characteristic function $\widehat{v_{n}^{2 \beta}}(\boldsymbol{\xi}, t)$ is given by

$$
v_{n}^{2 \beta}(\boldsymbol{x}, t)=v_{n}^{2 \beta}\left(x_{1}, x_{2}, \cdots, x_{n}, t\right)=\frac{1}{(2 \pi)^{n}} \int_{\mathbb{R}^{n}} e^{-i \boldsymbol{\xi} \cdot \boldsymbol{x}} e^{-t\|\boldsymbol{\xi}\|^{2 \beta}} d \boldsymbol{\xi} .
$$

The equation governing the distribution $v_{n}^{2 \beta}(\boldsymbol{x}, t)$ of the vector process $\boldsymbol{S}_{n}^{2 \beta}(t)$, $t>0$, is

$$
\left(\frac{\partial}{\partial t}+(-\Delta)^{\beta}\right) v_{n}^{2 \beta}(\boldsymbol{x}, t)=0, \quad \boldsymbol{x} \in \mathbb{R}^{n}, t>0,
$$


where the fractional negative Laplacian is related to the classical Laplacian by means of the following relationships (Bochner representation, see for example Balakrishnan [3]; Bochner [5])

$$
\begin{aligned}
& \frac{\sin \pi \beta}{\pi} \int_{0}^{\infty} d \lambda \lambda^{\beta-1}(\lambda-\Delta)^{-1} \Delta=\frac{\sin \pi \beta}{\pi} \int_{0}^{\infty} \lambda^{\beta-1}\left(\int_{0}^{\infty} e^{-w(\lambda-\Delta)} d w\right) \Delta d \lambda \\
= & \frac{\sin \pi \beta}{\pi} \Delta \Gamma(\beta) \int_{0}^{\infty} w^{1-\beta-1} e^{-w(-\Delta)} d w=\frac{\Delta}{\Gamma(1-\beta)} \int_{0}^{\infty} w^{1-\beta-1} e^{-w(-\Delta)} d w \\
= & -(-\Delta)^{\beta} .
\end{aligned}
$$

A definition of the fractional negative Laplacian can be given in the space of the Fourier transforms as follows

$$
-(-\Delta)^{\beta} u(\boldsymbol{x})=-\frac{1}{(2 \pi)^{n}} \int_{\mathbb{R}^{n}} e^{-i \boldsymbol{x} \cdot \boldsymbol{\xi}}\left(\xi_{1}^{2}+\xi_{2}^{2}+\cdots+\xi_{n}^{2}\right)^{\beta} \widehat{u}(\boldsymbol{\xi}) d \boldsymbol{\xi},
$$

where

$$
\operatorname{Dom}(-\Delta)^{\beta}=\left\{u \in L_{\mathrm{loc}}^{1}\left(\mathbb{R}^{n}\right): \int_{\mathbb{R}^{n}}|\widehat{u}(\boldsymbol{\xi})|^{2}\left(1+\|\boldsymbol{\xi}\|^{2 \beta}\right) d \boldsymbol{\xi}<\infty\right\} .
$$

An equivalent alternative definition of the $n$-dimensional fractional Laplacian is

$$
(-\Delta)^{\beta} u(\boldsymbol{x})=c(\beta, n) \text { P.V. } \int_{\mathbb{R}^{n}} \frac{u(\boldsymbol{x})-u(\boldsymbol{y})}{\|\boldsymbol{x}-\boldsymbol{y}\|^{n+2 \beta}} d y,
$$

where the multiplicative constant $c(\beta, n)$ must be evaluated in such a way that

$$
\int_{\mathbb{R}^{n}} e^{i \boldsymbol{\xi} \cdot \boldsymbol{x}}(-\Delta)^{\beta} u(\boldsymbol{x}) d \boldsymbol{x}=\|\boldsymbol{\xi}\|^{2 \beta} \int_{\mathbb{R}^{n}} e^{i \boldsymbol{\xi} \cdot \boldsymbol{x}} u(\boldsymbol{x}) d \boldsymbol{x} .
$$

Let us focus our attention on the one-dimensional case of (3.8). In this case we have that, for $0<2 \beta<1$,

$$
\begin{aligned}
& \left(-\frac{\partial^{2}}{\partial x^{2}}\right)^{\beta} u(x)=c(\beta, 1) \mathrm{P} . \mathrm{V} . \int_{\mathbb{R}} \frac{u(x)-u(y)}{|x-y|^{1+2 \beta}} \\
= & c(\beta, 1) \lim _{\epsilon \rightarrow 0}\left[\int_{-\infty}^{0-\epsilon} \frac{u(x)-u(x-z)}{|z|^{1+2 \beta}} d z+\int_{0+\epsilon}^{\infty} \frac{u(x)-u(x-z)}{|z|^{1+2 \beta}} d z\right] \\
= & c(\beta, 1) \lim _{\epsilon \rightarrow 0}\left[\int_{0+\epsilon}^{\infty} \frac{u(x)-u(x+z)}{z^{1+2 \beta}} d z+\int_{0+\epsilon}^{\infty} \frac{u(x)-u(x-z)}{z^{1+2 \beta}} d z\right] \\
= & \frac{\Gamma(1-2 \beta)}{2 \beta} c(\beta, 1)\left[\frac{1}{\Gamma(1-2 \beta)} \frac{d}{d x}\left(\int_{-\infty}^{x} \frac{u(z) d z}{(x-z)^{2 \beta}}-\int_{x}^{\infty} \frac{u(z) d z}{(z-x)^{2 \beta}}\right)\right],
\end{aligned}
$$

where in the intermediate steps, we considered the relation between the Marchaud and the Weyl fractional derivatives. By setting

$$
c(\beta, 1)=\frac{2 \beta}{2 \Gamma(1-2 \beta) \cos \beta \pi},
$$

we have that, for $0<2 \beta<1$,

$$
\begin{aligned}
& -\left(-\frac{\partial^{2}}{\partial x^{2}}\right)^{\beta} u(x)= \\
= & -\frac{1}{2 \cos \beta \pi}\left[\frac{1}{\Gamma(1-2 \beta)} \frac{d}{d x} \int_{-\infty}^{x} \frac{u(z) d z}{(x-z)^{2 \beta}}-\frac{1}{\Gamma(1-2 \beta)} \frac{d}{d x} \int_{x}^{\infty} \frac{u(z) d z}{(z-x)^{2 \beta}}\right]
\end{aligned}
$$




$$
=-\frac{1}{2 \cos \beta \pi} \frac{1}{\Gamma(1-2 \beta)} \frac{d}{d x} \int_{-\infty}^{\infty} \frac{u(z)}{|x-z|^{2 \beta}} d z=\frac{\partial^{2 \beta}}{\partial|x|^{2 \beta}} u(x),
$$

where $\frac{\partial^{2 \beta}}{\partial|x|^{2 \beta}}$ represents the Riesz operator.

Remark 3.1. We notice that, for $0<2 \beta<1$,

$$
\mathcal{F}\left[\frac{\partial^{2 \beta}}{\partial|x|^{2 \beta}} u(x)\right](\xi)=-|\xi|^{2 \beta} \widehat{u}(\xi) .
$$

This is due to the calculation

$$
\begin{aligned}
& \mathcal{F}\left[\frac{\partial^{2 \beta}}{\partial|x|^{2 \beta}} u(x)\right](\xi)= \\
= & -\frac{1}{2 \cos \beta \pi} \frac{1}{\Gamma(1-2 \beta)}\left[\int_{-\infty}^{\infty} d x e^{i \xi x}\left(\frac{d}{d x} \int_{-\infty}^{x} \frac{u(z) d z}{(x-z)^{2 \beta}}-\frac{d}{d x} \int_{x}^{\infty} \frac{u(z) d z}{(z-x)^{2 \beta}}\right)\right] \\
= & \frac{i \xi}{2 \cos \beta \pi} \frac{1}{\Gamma(1-2 \beta)}\left[\int_{-\infty}^{\infty} d x e^{i \xi x}\left(\int_{-\infty}^{x} \frac{u(z) d z}{(x-z)^{2 \beta}}-\int_{x}^{\infty} \frac{u(z) d z}{(z-x)^{2 \beta}}\right)\right] \\
= & \frac{i \xi}{2 \cos \beta \pi} \frac{1}{\Gamma(1-2 \beta)}\left[\int_{-\infty}^{\infty} d z u(z)\left(\int_{z}^{\infty} \frac{e^{i \xi x} d x}{(x-z)^{2 \beta}}-\int_{-\infty}^{z} \frac{e^{i \xi x} d x}{(z-x)^{2 \beta}}\right)\right] \\
= & \frac{i \xi}{2 \cos \beta \pi} \frac{1}{\Gamma(1-2 \beta)}\left[\int_{-\infty}^{\infty} e^{i \xi z} u(z) d z\left(\int_{0}^{\infty} \frac{e^{i \xi y}}{y^{2 \beta}} d y-\int_{0}^{\infty} \frac{e^{-i \xi y}}{y^{2 \beta}} d y\right)\right] \\
= & -\frac{2 \xi}{2 \cos \beta \pi} \frac{1}{\Gamma(1-2 \beta)} \int_{-\infty}^{\infty} e^{i \xi z} u(z) d z \int_{0}^{\infty} \frac{\sin \xi y}{y^{2 \beta}} d y \\
= & -\frac{\xi}{\cos \beta \pi} \frac{1}{\Gamma(1-2 \beta)} \frac{\widehat{u}(\xi)}{\Gamma(2 \beta)} \int_{0}^{\infty} \int_{0}^{\infty} \sin _{\xi y} e^{-w y} w^{2 \beta-1} d w d y \\
= & -\frac{\xi}{\cos \beta \pi} \frac{1}{\Gamma(1-2 \beta)} \frac{\widehat{u}(\xi)}{\Gamma(2 \beta)} \int_{0}^{\infty} d w w^{2 \beta-1} \int_{0}^{\infty} d y e^{-w y}\left(\frac{e^{i \xi y}-e^{-i \xi y}}{2 i}\right) \\
= & -\frac{\xi^{2}}{\cos \beta \pi} \frac{1}{\Gamma(1-2 \beta)} \frac{\widehat{u}(\xi)}{\Gamma(2 \beta)} \int_{0}^{\infty} d w \frac{w^{2 \beta-1}}{w^{2}+\xi^{2}} \\
= & -\frac{\xi^{2}}{\cos \beta \pi} \frac{1}{\Gamma(1-2 \beta)} \frac{\widehat{u}(\xi)}{\Gamma(2 \beta)} \int_{0}^{\infty} d w w^{2 \beta-1} \int_{0}^{\infty} d y e^{-y\left(w^{2}+\xi^{2}\right)} \\
= & -\frac{\xi^{2}}{2 \cos \beta \pi} \frac{1}{\Gamma(1-2 \beta)} \frac{\widehat{u}(\xi)}{\Gamma(2 \beta)} \frac{\Gamma(\beta) \Gamma(1-\beta)}{|\xi|^{2-2 \beta}}=-|\xi|^{2 \beta} \widehat{u}(\xi) .
\end{aligned}
$$

This concludes the proof of (3.13).

\section{Space-time fractional telegraph equation}

We consider now the composition of an isotropic vector of stable processes $\boldsymbol{S}_{n}^{2 \beta}(t)$, $t>0$, defined in (3.1), with the positively-valued process, defined in (2.19),

$$
\mathcal{L}^{\nu}(t)=\inf \left\{s>0: \mathcal{H}^{\nu}(s)=H_{1}^{2 \nu}(s)+(2 \lambda)^{\frac{1}{\nu}} H_{2}^{\nu}(s) \geq t\right\}, \quad t>0,
$$

where $H_{1}^{2 \nu}, H_{2}^{\nu}$ are independent positively skewed stable processes of order $2 \nu$ and $\nu$, respectively. The distribution $w_{\nu}^{\beta}(\boldsymbol{x}, t)$ of the process $\boldsymbol{S}_{n}^{2 \beta}\left(c^{2} \mathcal{L}^{\nu}(t)\right), t>0, \beta \in$ 
$(0,1]$, is the fundamental solution to the space-time fractional telegraph equation

$$
\left(\frac{{ }^{C} \partial^{2 \nu}}{\partial t^{2 \nu}}+2 \lambda \frac{{ }^{C} \partial^{\nu}}{\partial t^{\nu}}\right) w_{\nu}^{\beta}(\boldsymbol{x}, t)=-c^{2}(-\Delta)^{\beta} w_{\nu}^{\beta}(\boldsymbol{x}, t), \quad \boldsymbol{x} \in \mathbb{R}^{n}, t>0 .
$$

In our view the next theorem generalizes some previous results because we here have fractionality in space and time and the equation (4.2) is defined in $\mathbb{R}^{n}$.

Theorem 4.1. For $\nu \in\left(0, \frac{1}{2}\right], \beta \in(0,1]$ and $c>0$ the solution to the Cauchy problem for the space-time fractional $n$-dimensional telegraph equation

$$
\left\{\begin{array}{l}
\left(\frac{C}{\partial t^{2 \nu}}+2 \lambda \frac{C}{\partial t^{\nu}}\right) w_{\nu}^{\beta}(\boldsymbol{x}, t)=-c^{2}(-\Delta)^{\beta} w_{\nu}^{\beta}(\boldsymbol{x}, t), \quad \boldsymbol{x} \in \mathbb{R}^{n}, t>0 \\
w_{\nu}^{\beta}(\boldsymbol{x}, 0)=\delta(\boldsymbol{x}),
\end{array}\right.
$$

coincides with the probability law of the vector process

$$
\boldsymbol{W}_{n}(t)=\boldsymbol{S}_{n}^{2 \beta}\left(c^{2} \mathcal{L}^{\nu}(t)\right), \quad t>0
$$

and has Fourier transform which reads

$$
\begin{aligned}
& \widehat{w_{\nu}^{\beta}}(\boldsymbol{\xi}, t)= \\
= & \frac{1}{2}\left[\left(1+\frac{\lambda}{\sqrt{\lambda^{2}-c^{2}\|\boldsymbol{\xi}\|^{2 \beta}}}\right) E_{\nu, 1}\left(r_{1} t^{\nu}\right)+\left(1-\frac{\lambda}{\sqrt{\lambda^{2}-c^{2}\|\boldsymbol{\xi}\|^{2 \beta}}}\right) E_{\nu, 1}\left(r_{2} t^{\nu}\right)\right],
\end{aligned}
$$

where

$$
r_{1}=-\lambda+\sqrt{\lambda^{2}-c^{2}\|\boldsymbol{\xi}\|^{2 \beta}}, \quad r_{2}=-\lambda-\sqrt{\lambda^{2}-c^{2}\|\boldsymbol{\xi}\|^{2 \beta}} .
$$

The time derivatives appearing in (4.3) must be meant in the Dzerbayshan-Caputo sense. The fractional Laplacian is defined in (3.6).

Proof. By taking the Laplace transform of (4.3) we have

$\mu^{2 \nu} \widetilde{w_{\nu}^{\beta}}(\boldsymbol{x}, \mu)-\mu^{2 \nu-1} \delta(\boldsymbol{x})+2 \lambda\left[\mu^{\nu} \widetilde{w_{\nu}^{\beta}}(\boldsymbol{x}, \mu)-\mu^{\nu-1} \delta(\boldsymbol{x})\right]=-c^{2}(-\Delta)^{\beta} \widetilde{w_{\nu}^{\beta}}(\boldsymbol{x}, \mu)$,

where we used the fact that (see [11] page 98, Lemma 2.24)

$$
\mathcal{L}\left[\frac{{ }^{C} \partial^{\nu}}{\partial t^{\nu}} w_{\nu}^{\beta}(\boldsymbol{x}, t)\right]=\mu^{\nu} \widetilde{w_{\nu}^{\beta}}(\boldsymbol{x}, \mu)-\mu^{\nu-1} w_{\nu}^{\beta}(\boldsymbol{x}, 0) .
$$

Now the Fourier transform of (4.7) yields

$$
\left(\mu^{2 \nu}+2 \lambda \mu^{\nu}\right) \widehat{\widehat{w_{\nu}^{\beta}}}(\boldsymbol{\xi}, \mu)-\left(\mu^{2 \nu-1}+2 \lambda \mu^{\nu-1}\right)=-c^{2}\|\boldsymbol{\xi}\|^{2 \beta} \widehat{\widehat{w_{\nu}^{\beta}}}(\boldsymbol{\xi}, \mu),
$$

and thus

$$
\widehat{\widehat{w_{\nu}^{\beta}}}(\boldsymbol{\xi}, \mu)=\frac{\mu^{2 \nu-1}+2 \lambda \mu^{\nu-1}}{\mu^{2 \nu}+2 \lambda \mu^{\nu}+c^{2}\|\boldsymbol{\xi}\|^{2 \beta}}, \quad \mu>0, \boldsymbol{\xi} \in \mathbb{R}^{n} .
$$

The probability density of the process $\boldsymbol{W}_{n}(t), t>0$, defined in (4.4), can be written as

$$
w_{\nu}^{\beta}(\boldsymbol{x}, t)=\int_{0}^{\infty} v_{\beta}\left(\boldsymbol{x}, c^{2} s\right) \iota_{\nu}(s, t) d s
$$

and has Fourier transform equal to

$$
\int_{\mathbb{R}^{n}} e^{i \boldsymbol{\xi} \cdot \boldsymbol{x}} w_{\nu}^{\beta}(\boldsymbol{x}, t) d \boldsymbol{x}=\int_{0}^{\infty} e^{-c^{2} s\|\boldsymbol{\xi}\|^{2 \beta}} c_{\nu}(s, t) d s .
$$


In order to show that the Laplace transform of (4.12) concides with (4.10), we have to derive the Laplace transform of $\varepsilon_{\nu}(x, t)$, with respect to the time $t$. Since

$$
\operatorname{Pr}\left\{\mathcal{L}^{\nu}(t)<x\right\}=\operatorname{Pr}\left\{\mathcal{H}^{\nu}(x)>t\right\}
$$

we have that

$$
\begin{aligned}
& \widetilde{L}_{\nu}(x, \mu)= \\
= & \int_{0}^{\infty} e^{-\mu t} \frac{\partial}{\partial x} \int_{t}^{\infty} \operatorname{Pr}\left\{\mathcal{H}^{\nu}(x) \in d s\right\} d t=\int_{0}^{\infty} e^{-\mu t}\left(-\frac{\partial}{\partial x} \int_{0}^{t} \hbar_{\nu}(s, x) d s\right) d t \\
= & -\frac{\partial}{\partial x} \frac{e^{-x \mu^{2 \nu}-2 \lambda x \mu^{\nu}}}{\mu}=\left(\mu^{2 \nu-1}+2 \lambda \mu^{\nu-1}\right) e^{-x \mu^{2 \nu}-2 \lambda x \mu^{\nu}}
\end{aligned}
$$

where we used result (2.28). Now we can complete the proof by taking the Laplace transform of (4.12) so that, in view of (4.14), we obtain

$$
\begin{aligned}
& \int_{0}^{\infty} e^{-\mu t} d t \int_{0}^{\infty} e^{-c^{2} s\|\boldsymbol{\xi}\|^{2 \beta}} L_{\nu}(s, t) d s= \\
= & \left(\mu^{2 \nu-1}+2 \lambda \mu^{\nu-1}\right) \int_{0}^{\infty} e^{-s c^{2}\|\boldsymbol{\xi}\|^{2 \beta}-s \mu^{2 \nu}-2 \lambda s \mu^{\nu}} d s=\frac{\mu^{2 \nu-1}+2 \lambda \mu^{\nu-1}}{\mu^{2 \nu}+2 \lambda \mu^{\nu}+c^{2}\|\boldsymbol{\xi}\|^{2 \beta}},
\end{aligned}
$$

which coincides with (4.10). The unicity of Fourier-Laplace transform proves that the claimed result holds. The proof that the Fourier transform of $w_{\nu}^{\beta}(\boldsymbol{x}, t)$ has the form (4.5) can be carried out by means of the calculation performed in Theorem 2.2. We have that

$$
\begin{aligned}
\widehat{\widetilde{w_{\nu}^{\beta}}}(\boldsymbol{\xi}, \mu) & =\frac{\mu^{2 \nu-1}+2 \lambda \mu^{\nu-1}}{\mu^{2 \nu}+2 \lambda \mu^{\nu}+c^{2}\|\boldsymbol{\xi}\|^{2 \beta}}=\frac{\mu^{\nu-1}}{\mu^{\nu}-r_{1}}+\frac{\mu^{\nu-1}}{\mu^{\nu}-r_{2}}-\frac{\mu^{2 \nu-1}}{\left(\mu^{\nu}-r_{1}\right)\left(\mu^{\nu}-r_{2}\right)} \\
& =\frac{\mu^{\nu-1}}{\mu^{\nu}-r_{1}}+\frac{\mu^{\nu-1}}{\mu^{\nu}-r_{2}}-\left[\frac{\mu^{\nu-(1-\nu)}}{\mu^{\nu}-r_{1}}-\frac{\mu^{\nu-(1-\nu)}}{\mu^{\nu}-r_{2}}\right] \frac{1}{2 \sqrt{\lambda^{2}-c^{2}\|\boldsymbol{\xi}\|^{2 \beta}}},
\end{aligned}
$$

where

$$
r_{1}=-\lambda+\sqrt{\lambda^{2}-c^{2}\|\boldsymbol{\xi}\|^{2 \beta}}, \quad r_{2}=-\lambda-\sqrt{\lambda^{2}-c^{2}\|\boldsymbol{\xi}\|^{2 \beta}} .
$$

and thus by inverting (4.16) by means of (2.33), we obtain result (4.5). An alternative derivation of (4.5) can be carried out as follows

$$
\begin{aligned}
\widehat{w_{\nu}^{\beta}}(\boldsymbol{\xi}, t) & =\int_{-\infty}^{\infty} e^{i \boldsymbol{\xi} \cdot \boldsymbol{x}} d \boldsymbol{x} \int_{0}^{\infty} \operatorname{Pr}\left\{\boldsymbol{S}_{n}^{2 \beta}\left(c^{2} s\right) \in d \boldsymbol{x}\right\} \operatorname{Pr}\left\{\mathcal{L}^{\nu}(t) \in d s\right\} \\
& =\int_{0}^{\infty} e^{-c^{2} s\|\boldsymbol{\xi}\|^{2 \beta}} \operatorname{Pr}\left\{\mathcal{L}^{\nu}(t) \in d s\right\}=(4.5)
\end{aligned}
$$

because of Theorem 2.2 .

4.1. The case $\nu=\frac{1}{2}$, subordinator with drift. The fractional equation (4.2), for $n=1, \nu=\frac{1}{2}$, reads

$$
\left(\frac{\partial}{\partial t}+2 \lambda \frac{{ }^{C} \partial^{\frac{1}{2}}}{\partial t^{\frac{1}{2}}}\right) w_{\frac{1}{2}}^{\beta}(x, t)=c^{2}\left(\frac{\partial^{2 \beta}}{\partial|x|^{2 \beta}}\right) w_{\frac{1}{2}}^{\beta}(x, t), \quad 0<\beta<1,
$$


where $\frac{\partial^{2 \beta}}{\partial|x|^{2 \beta}}$ is the Riesz operator defined in (3.12). For $\beta=1$ we have the special case

$$
\left(\frac{\partial}{\partial t}+2 \lambda \frac{{ }^{C} \partial^{\frac{1}{2}}}{\partial t^{\frac{1}{2}}}\right) w_{\frac{1}{2}}^{1}(x, t)=c^{2} \frac{\partial^{2}}{\partial x^{2}} w_{\frac{1}{2}}^{1}(x, t)
$$

dealt with in Orsingher and Beghin [13]. The construction of the composition related to equation (4.19) involves the subordinator

$$
\mathcal{H}^{\frac{1}{2}}(t)=t+(2 \lambda)^{2} H^{\frac{1}{2}}(t), \quad t>0,
$$

where $H^{\frac{1}{2}}(t), t>0$, is a positively-skewed stable process and has the same law as the first-passage time of a Brownian motion through level $\frac{t}{\sqrt{2}}$. We note that $\mathcal{H}^{\frac{1}{2}}(t)$, $t>0$, has distribution with support $[t, \infty)$ and thus differs from $\mathcal{H}^{\nu}(t), t>0$, $0<\nu<\frac{1}{2}$, which instead has support $[0, \infty)$. The distribution of $(4.21)$ writes

$$
\operatorname{Pr}\left\{\mathcal{H}^{\frac{1}{2}}(t)<x\right\}=\int_{0}^{\frac{x-t}{(2 \lambda)^{2}}} \frac{t}{\sqrt{2}} \frac{e^{-\frac{t^{2}}{4 z}}}{\sqrt{2 \pi z^{3}}} d z, \quad x>t>0 .
$$

The inverse process

$$
\mathcal{L}^{\frac{1}{2}}(t)=\inf \left\{s: s+(2 \lambda)^{2} H^{\frac{1}{2}}(s) \geq t\right\}=\inf \left\{s: \mathcal{H}^{\frac{1}{2}}(s) \geq t\right\}
$$

is related to (4.21) by means of the relationship

$$
\operatorname{Pr}\left\{\mathcal{L}^{\frac{1}{2}}(t)<x\right\}=\operatorname{Pr}\left\{\mathcal{H}^{\frac{1}{2}}(x)>t\right\}=\int_{\frac{t-x}{(2 \lambda)^{2}}}^{\infty} \frac{x}{\sqrt{2}} \frac{e^{-\frac{x^{2}}{4 z}}}{\sqrt{2 \pi z^{3}}} d z .
$$

From (4.24) we can extract the distributon of $\mathcal{L}^{\frac{1}{2}}(t), t>0$, in the following manner

$$
\begin{aligned}
\mathfrak{l}_{\frac{1}{2}}(x, t) & =\frac{\operatorname{Pr}\left\{\mathcal{L}^{\frac{1}{2}}(t) \in d x\right\}}{d x}=\frac{\partial}{\partial x} \int_{\frac{t-x}{(2 \lambda)^{2}}}^{\infty} \frac{x e^{-\frac{x^{2}}{4 z}}}{\sqrt{4 \pi z^{3}}} d z \\
& =\frac{2 \lambda x e^{-\frac{(2 \lambda x)^{2}}{4(t-x)}}}{\sqrt{4 \pi(t-x)^{3}}}+2 \lambda \frac{e^{-\frac{(2 \lambda x)^{2}}{4(t-x)}}}{\sqrt{\pi(t-x)}}, \quad 0<x<t .
\end{aligned}
$$

Remark 4.1. The distribution (4.25) can be also obtained from the general case (2.50) which for $\nu=\frac{1}{2}$ becomes, for $0<x<t$,

$$
\begin{aligned}
\mathfrak{l}_{\frac{1}{2}}(x, t) & =\int_{0}^{t} \delta(s-x) h_{\frac{1}{2}}(t-s, 2 \lambda x) d s+2 \lambda \int_{0}^{t} l_{\frac{1}{2}}(2 \lambda x, s) \delta(x-(t-s)) d s \\
& =h_{\frac{1}{2}}(t-x, 2 \lambda x)+2 \lambda l_{\frac{1}{2}}(2 \lambda x, t-x) \\
& =\frac{2 \lambda x e^{-\frac{(2 \lambda x)^{2}}{4(t-x)}}}{\sqrt{4 \pi(t-x)^{3}}}+2 \lambda \frac{e^{-\frac{(2 \lambda x)^{2}}{4(t-x)}}}{\sqrt{\pi(t-x)}}
\end{aligned}
$$

In the last step we used the fact that

$$
L^{\frac{1}{2}}(t) \stackrel{l a w}{=}|B(t)|, \quad t>0,
$$

where $L^{\frac{1}{2}}(t), t>0$, dealt with in section 1.3 , is the inverse of the totally positivelyskewed stable process $H^{\frac{1}{2}}(t), t>0$. 
The $t$-Laplace transform of (4.25) becomes

$$
\begin{aligned}
\widetilde{\mathfrak{l}_{\frac{1}{2}}}(x, \mu) & =\int_{x}^{\infty} e^{-\mu t} \mathfrak{l}_{\frac{1}{2}}(x, t) d t= \\
& =\frac{2 \lambda x}{\sqrt{2}} \int_{x}^{\infty} e^{-\mu t} \frac{e^{-\frac{(2 \lambda x)^{2}}{4(t-x)}}}{\sqrt{2 \pi(t-x)^{3}}} d t+2 \lambda \int_{x}^{\infty} e^{-\mu t} \frac{e^{-\frac{(2 \lambda x)^{2}}{4(t-x)}}}{\sqrt{\pi(t-x)}} d t \\
& =\frac{2 \lambda x}{\sqrt{2}} e^{-\mu x} \int_{0}^{\infty} e^{-\mu t} \frac{e^{-\frac{(2 \lambda x)^{2}}{4 t}}}{\sqrt{2 \pi t^{3}}} d t+2 \lambda e^{-\mu x} \int_{0}^{\infty} e^{-\mu t} \frac{e^{-\frac{(2 \lambda x)^{2}}{4 t}}}{\sqrt{\pi t}} d t \\
& =e^{-\mu x} e^{-2 \lambda x \sqrt{\mu}}+2 \lambda \mu^{-\frac{1}{2}} e^{-\mu x} e^{-2 \lambda x \sqrt{\mu}} .
\end{aligned}
$$

Finally the $x$-Laplace transform of (4.28) becomes

$$
\begin{aligned}
\widetilde{\widetilde{c}_{\frac{1}{2}}}(\gamma, \mu) & =\int_{0}^{\infty} e^{-\gamma x}\left(\int_{x}^{\infty} e^{-\mu t} l_{\frac{1}{2}}(x, t) d t\right) d x \\
& =\frac{1}{\mu+\gamma+2 \lambda \sqrt{\mu}}+\frac{2 \lambda}{\sqrt{\mu}} \frac{1}{\mu+\gamma+2 \lambda \sqrt{\mu}}=\frac{1+2 \lambda \mu^{-\frac{1}{2}}}{\mu+\gamma+2 \lambda \sqrt{\mu}},
\end{aligned}
$$

which coincides with $(2.31)$, for $\nu=\frac{1}{2}$. Let us now consider the process $\boldsymbol{W}_{n}(t)=$ $\boldsymbol{S}_{n}^{2 \beta}\left(c^{2} \mathcal{L}^{\nu}(t)\right), t>0$, dealt with in Theorem 4.1. For $\beta=1, n=1$ and $\nu=\frac{1}{2}$ this process becomes

$$
W_{1}(t)=S_{1}^{2}\left(c^{2} \mathcal{L}^{\frac{1}{2}}(t)\right)=B\left(c^{2} \mathcal{L}^{\frac{1}{2}}(t)\right), \quad t>0
$$

where $B$ represents a standard Brownian motion and $\mathcal{L}^{\frac{1}{2}}(t), t>0$, is the process defined in (4.23). With

$$
p_{|B|}(x, t)=\frac{e^{-\frac{x^{2}}{4 t}}}{\sqrt{\pi t}}, \quad x>0, t>0,
$$

we denote the law of the process $|B(t)|, t>0$. In view of the previous results we are able to prove the following theorem.

Theorem 4.2. The law of (4.30) coincides with the law of the composition

$$
\mathcal{W}(t)=T(|B(t)|), \quad t>0
$$

where $T$ is the telegraph process (1.20) with parameters $c>0, \lambda>0$ and law $p_{T}(x, t)$ which has characteristic function

$$
\begin{aligned}
& \widehat{p_{T}}(\xi, t)= \\
= & \frac{1}{2}\left[\left(1+\frac{\lambda}{\sqrt{\lambda^{2}-c^{2} \xi^{2}}}\right) e^{-\lambda t+t \sqrt{\lambda^{2}-c^{2} \xi^{2}}}+\left(1-\frac{\lambda}{\sqrt{\lambda^{2}-c^{2} \xi^{2}}}\right) e^{-\lambda t-t \sqrt{\lambda^{2}-c^{2} \xi^{2}}}\right] .
\end{aligned}
$$

In other words we have the following equality in distribution

$$
B\left(c^{2} \mathcal{L}^{\frac{1}{2}}(t)\right) \stackrel{\text { law }}{=} T(|B(t)|), \quad t>0
$$

Proof. First we show that the Fourier-Laplace transform of the law $w_{\frac{1}{2}}^{1}(x, t)$ of the process $W_{1}(t)=S_{1}^{2}\left(c^{2} \mathcal{L}^{\frac{1}{2}}(t)\right)=B\left(c^{2} \mathcal{L}^{\frac{1}{2}}(t)\right), t>0$, is written as in (4.15) for 
$\nu=\frac{1}{2}, \beta=1, n=1$, and reads

$$
\widehat{\widetilde{w_{\frac{1}{2}}^{1}}}(\xi, \mu)=\frac{1+2 \lambda \mu^{-\frac{1}{2}}}{\mu+2 \lambda \sqrt{\mu}+c^{2} \xi^{2}} .
$$

We have that

$$
\begin{aligned}
\widetilde{w_{\frac{1}{2}}^{1}}(x, \mu) & =\int_{0}^{\infty} e^{-\mu t}\left(\int_{0}^{t} p_{B}\left(x, c^{2} s\right) l_{\frac{1}{2}}(s, t) d s\right) d t \\
& =\int_{0}^{\infty} p_{B}\left(x, c^{2} s\right) d s \int_{s}^{\infty} e^{-\mu t} l_{\frac{1}{2}}(s, t) d t \\
& =\int_{0}^{\infty} p_{B}\left(x, c^{2} s\right) d s\left[\int_{s}^{\infty} e^{-\mu t}\left(\frac{2 \lambda s e^{-\frac{(2 \lambda s)^{2}}{4(t-s)}}}{\sqrt{4 \pi(t-s)^{3}}}+2 \lambda \frac{e^{-\frac{(2 \lambda s)^{2}}{4(t-s)}}}{\sqrt{\pi(t-s)}}\right) d t\right] \\
& =\int_{0}^{\infty} p_{B}\left(x, c^{2} s\right)\left(e^{-s(\mu+2 \lambda \sqrt{\mu})}+2 \lambda \sqrt{\mu} e^{-s(\mu+2 \lambda \sqrt{\mu})}\right) d s \\
& =\int_{0}^{\infty} \frac{e^{-\frac{x^{2}}{4 c^{2} s}}}{\sqrt{4 \pi c^{2} s}} e^{-s(\mu+2 \lambda \sqrt{\mu})} d s+2 \lambda \mu^{-\frac{1}{2}} \int_{0}^{\infty} \frac{e^{-\frac{x^{2}}{4 c^{2} s}}}{\sqrt{4 \pi c^{2} s}} e^{-s(\mu+2 \lambda \sqrt{\mu})} d s,
\end{aligned}
$$

and thus taking the Fourier transform we get

$$
\begin{aligned}
\widehat{\widetilde{w_{\frac{1}{2}}^{1}}}(\xi, \mu) & =\int_{0}^{\infty} e^{-s c^{2} \xi^{2}} e^{-s(\mu+2 \lambda \sqrt{\mu})} d s+2 \lambda \mu^{-\frac{1}{2}} \int_{0}^{\infty} e^{-s c^{2} \xi^{2}} e^{-s(\mu+2 \lambda \sqrt{\mu})} d s \\
& =\frac{1+2 \lambda \mu^{-\frac{1}{2}}}{\mu+2 \lambda \sqrt{\mu}+c^{2} \xi^{2}} .
\end{aligned}
$$

Now we are going to prove that the law $w(x, t)$ of the process $\mathcal{W}(t), t>0$, has Fourier-Laplace transform that coincides with (4.35). We have that

$$
w(x, t)=\int_{0}^{\infty} p_{T}(x, s) p_{|B|}(s, t) d s,
$$

and thus the Fourier transform of $w(x, t)$ reads

$$
\begin{aligned}
\widehat{w}(\xi, t)= & \int_{-\infty}^{\infty} e^{i \xi x} d x \int_{0}^{\infty} p_{T}(x, s) p_{|B|}(s, t) d s \\
= & \frac{1}{2} \int_{0}^{\infty}\left[\left(1+\frac{\lambda}{\sqrt{\lambda^{2}-c^{2} \xi^{2}}}\right) e^{-\lambda s+s \sqrt{\lambda^{2}-c^{2} \xi^{2}}}\right. \\
& \left.+\left(1-\frac{\lambda}{\sqrt{\lambda^{2}-c^{2} \xi^{2}}}\right) e^{-\lambda s-s \sqrt{\lambda^{2}-c^{2} \xi^{2}}}\right] p_{|B|}(s, t) d s .
\end{aligned}
$$

Passing now to the Laplace transform we have

$$
\begin{aligned}
\widetilde{\widehat{w}}(\xi, \mu)= & \frac{1}{2} \int_{0}^{\infty} e^{-\mu t} d t \int_{0}^{\infty}\left[\left(1+\frac{\lambda}{\sqrt{\lambda^{2}-c^{2} \xi^{2}}}\right) e^{-\lambda s+s \sqrt{\lambda^{2}-c^{2} \xi^{2}}}\right. \\
& \left.+\left(1-\frac{\lambda}{\sqrt{\lambda^{2}-c^{2} \xi^{2}}}\right) e^{-\lambda s-s \sqrt{\lambda^{2}-c^{2} \xi^{2}}}\right] \frac{e^{-\frac{s^{2}}{4 t}}}{\sqrt{\pi t}} d s
\end{aligned}
$$




$$
\begin{aligned}
= & \frac{1}{2} \int_{0}^{\infty}\left[\left(1+\frac{\lambda}{\sqrt{\lambda^{2}-c^{2} \xi^{2}}}\right) e^{-\lambda s+s \sqrt{\lambda^{2}-c^{2} \xi^{2}}}\right. \\
& \left.+\left(1-\frac{\lambda}{\sqrt{\lambda^{2}-c^{2} \xi^{2}}}\right) e^{-\lambda s-s \sqrt{\lambda^{2}-c^{2} \xi^{2}}}\right] \frac{e^{-s \sqrt{\mu}}}{\sqrt{\mu}} d s \\
= & \frac{1}{2 \sqrt{\mu}}\left[\left(1+\frac{\lambda}{\sqrt{\lambda^{2}-c^{2} \xi^{2}}}\right)\left(\frac{1}{\lambda+\sqrt{\mu}-\sqrt{\lambda^{2}-c^{2} \xi^{2}}}\right)\right. \\
& \left.+\left(1-\frac{\lambda}{\sqrt{\lambda^{2}-c^{2} \xi^{2}}}\right)\left(\frac{1}{\lambda+\sqrt{\mu}+\sqrt{\lambda^{2}-c^{2} \xi^{2}}}\right)\right] \\
= & \frac{\left(\lambda+\sqrt{\lambda^{2}-c^{2} \xi^{2}}\right)\left(\lambda+\sqrt{\mu}+\sqrt{\lambda^{2}-c^{2} \xi^{2}}\right)}{\left(2 \sqrt{\mu} \sqrt{\lambda^{2}-c^{2} \xi^{2}}\right)\left(\mu+2 \lambda \sqrt{\mu}+c^{2} \xi^{2}\right)} \\
& +\frac{\left(\sqrt{\lambda^{2}-c^{2} \xi^{2}}-\lambda\right)\left(\lambda+\sqrt{\mu}-\sqrt{\lambda^{2}-c^{2} \xi^{2}}\right)}{\left(2 \sqrt{\mu} \sqrt{\lambda^{2}-c^{2} \xi^{2}}\right)\left(\mu+2 \lambda \sqrt{\mu}+c^{2} \xi^{2}\right)} \\
= & \frac{1+2 \lambda \mu^{-\frac{1}{2}}}{\mu+2 \lambda \sqrt{\mu}+c^{2} \xi^{2}},
\end{aligned}
$$

which coincides with (4.35).

This shows that for each $t$ we have the following equality in distribution

$$
T(|B(t)|) \stackrel{\text { law }}{=} B\left(c^{2} \mathcal{L}^{\frac{1}{2}}(t)\right), \quad t>0,
$$

where the role of the Brownian motion is interchanged in the two members of (4.41). Thus, by suitably slowing down the time in (4.41), we obtain the same distributional effect of a telegraph process taken at a Brownian time.

Remark 4.2. The probability distribution of the process

$$
W_{1}(t)=B\left(c^{2} \mathcal{L}^{\frac{1}{2}}(t)\right), \quad t>0,
$$

can be written as

$$
\begin{aligned}
w_{\frac{1}{2}}^{1}(x, t) & =\frac{\lambda}{c \pi} \int_{0}^{t} \frac{1}{\sqrt{s(t-s)}} e^{-\frac{x^{2}}{4 c^{2} s}-\frac{\lambda^{2} s^{2}}{t-s}}\left[\frac{s}{2(t-s)}+1\right] d s \\
& =\frac{\lambda}{c \pi} \int_{0}^{t} \frac{1}{\sqrt{s(t-s)}} e^{-\frac{x^{2}}{4 c^{2} s}-\frac{\lambda^{2} s^{2}}{t-s}}\left[\frac{1}{2}\left(1+\frac{t}{t-s}\right)\right] d s \\
& \stackrel{y=\lambda s}{=} \frac{\sqrt{\lambda}}{c \pi} \int_{0}^{\lambda t} e^{-\frac{\lambda x^{2}}{4 c^{2} y}} e^{-\frac{y^{2}}{t-\frac{y}{\lambda}}} \frac{1}{\sqrt{y} \sqrt{t-\frac{y}{\lambda}}}\left[\frac{1}{2}\left(1+\frac{t}{t-\frac{y}{\lambda}}\right)\right] d y .
\end{aligned}
$$

Taking the limit for $c \rightarrow \infty, \lambda \rightarrow \infty, \frac{c^{2}}{\lambda} \rightarrow 1$, formula (4.43) becomes

$$
\lim _{\substack{\lambda, c \rightarrow \infty \\ \frac{c^{2}}{\lambda} \rightarrow 1}} y_{\frac{1}{2}}^{1}(x, t)=2 \int_{0}^{\infty} \frac{e^{-\frac{x^{2}}{4 y}}}{\sqrt{4 \pi y}} \frac{e^{-\frac{y^{2}}{t}}}{\sqrt{\pi t}} d y
$$


which coincides with the distribution of an iterated Brownian motion $B_{1}\left(\left|B_{2}(t)\right|\right)$, $t>0$, with $B_{j}, j=1,2$, independent Brownian motions. From (4.43) we can see that the distribution of $W_{1}(t), t>0$, has a bell-shaped structure.

Finally we show that the density $w_{\frac{1}{2}}^{1}(x, t)$ integrates to unity in force of the calculation

$$
\begin{aligned}
\int_{-\infty}^{\infty} w_{\frac{1}{2}}^{1}(x, t) d x & =\int_{-\infty}^{\infty} d x \int_{0}^{t} d s \frac{e^{-\frac{x^{2}}{4 s}}}{\sqrt{4 \pi s}} l_{\frac{1}{2}}(s, t)=\int_{0}^{t} d s\left(\frac{\partial}{\partial s} \int_{\frac{t-s}{(2 \lambda)^{2}}}^{\infty} \frac{s e^{-\frac{s^{2}}{4 z}}}{\sqrt{4 \pi z^{3}}} d z\right) \\
& =\left[\int_{\frac{t-s}{(2 \lambda)^{2}}}^{\infty} \frac{s e^{-\frac{s^{2}}{4 z}}}{\sqrt{4 \pi z^{3}}} d z\right]_{s=0}^{s=t}=\int_{0}^{\infty} \frac{t e^{-\frac{t^{2}}{4 z}}}{\sqrt{4 \pi z^{3}}} d z=1 .
\end{aligned}
$$

In the intermediate step, formula (4.25) has been applied.

Remark 4.3. The characteristic function of the process $T^{2 \beta}(t), t>0$, whose distribution satisfies

$$
\left\{\begin{array}{l}
\left(\frac{\partial^{2}}{\partial t^{2}}+2 \lambda \frac{\partial}{\partial t}\right) p_{T}^{2 \beta}(x, t)=c^{2} \frac{\partial^{2 \beta}}{\partial|x|^{2 \beta}} p_{T}^{2 \beta}(x, t), \quad 0<\beta<1, \beta \neq \frac{1}{2} \\
p_{T}^{2 \beta}(x, 0)=\delta(x) \\
\left.\frac{\partial}{\partial t} p_{T}^{2 \beta}(x, t)\right|_{t=0}=0
\end{array}\right.
$$

reads

$$
\begin{aligned}
& \mathbb{E} e^{i \xi T^{2 \beta}(t)}= \\
= & \frac{e^{-\lambda t}}{2}\left[\left(1+\frac{\lambda}{\sqrt{\lambda^{2}-c^{2}|\xi|^{2 \beta}}}\right) e^{t \sqrt{\lambda^{2}-c^{2}|\xi|^{2 \beta}}}+\left(1-\frac{\lambda}{\sqrt{\lambda^{2}-c^{2}|\xi|^{2 \beta}}}\right) e^{-t \sqrt{\lambda^{2}-c^{2}|\xi|^{2 \beta}}}\right]
\end{aligned}
$$

see Orsingher and Zhao [14]. Therefore by performing the same steps as in theorem (4.2) we prove that

$$
S_{1}^{2 \beta}\left(\mathcal{L}^{\frac{1}{2}}(t)\right) \stackrel{\text { law }}{=} T^{2 \beta}(|B(t)|), \quad t>0 .
$$

4.2. The case $\nu=\frac{1}{3}$, convolutions of Airy functions. We first recall that the totally positively-skewed stable process $H^{\frac{1}{3}}(t), t>0$ has law

$$
\operatorname{Pr}\left\{H^{\frac{1}{3}}(t) \in d x\right\}=\frac{t}{x \sqrt[3]{3 x}} \operatorname{Ai}\left(\frac{t}{\sqrt[3]{3 x}}\right) d x, \quad x>0, t>0,
$$

where $\operatorname{Ai}(\cdot)$ is the Airy function. Result (4.49) can be obtained from the general series expansion of the stable law of order $\frac{1}{3}$ (see Orsingher and Beghin [16] page 245) which reads

$$
\begin{aligned}
h_{\frac{1}{3}}(x, 1) & =\frac{1}{3 \pi} \sum_{k=0}^{\infty}(-1)^{k} \frac{\Gamma\left(\frac{k+1}{3}\right)}{k !} x^{-\frac{1}{3}(k+1)-1} \sin \left(\frac{\pi}{3}(k+1)\right) \\
& =\frac{1}{3 \pi} \sum_{k=0}^{\infty}(-1)^{k} \frac{\Gamma\left(\frac{k+1}{3}\right)}{k !} x^{-\frac{k+1}{3}-1}(-1)^{k} \sin \left(\frac{2 \pi(k+1)}{3}\right) \\
& =\frac{1}{3} \frac{3^{\frac{2}{3}}}{x \sqrt[3]{x}} \mathrm{Ai}\left(\frac{1}{\sqrt[3]{3 x}}\right)=\frac{1}{x \sqrt[3]{3 x}} \mathrm{Ai}\left(\frac{1}{\sqrt[3]{3 x}}\right)
\end{aligned}
$$


where we used formula (4.10) of [16], which reads

$$
\operatorname{Ai}(w)=\frac{3^{-\frac{2}{3}}}{\pi} \sum_{k=0}^{\infty}\left(3^{\frac{1}{3}} w\right)^{k} \frac{\sin \left(\frac{2 \pi(k+1)}{3}\right)}{k !} \Gamma\left(\frac{k+1}{3}\right) .
$$

Since

$$
H^{\frac{1}{3}}(t) \stackrel{\text { law }}{=} t^{3} H^{\frac{1}{3}}(1),
$$

we have result (4.49). From the relatioship between $H^{\frac{1}{3}}(t), t>0$, and the inverse process $L^{\frac{1}{3}}(t), t>0$,

$$
\operatorname{Pr}\left\{H^{\frac{1}{3}}(t)<x\right\}=\operatorname{Pr}\left\{L^{\frac{1}{3}}(x)>t\right\}
$$

we extract the density of $L^{\frac{1}{3}}(x), x>0$,

$$
\begin{aligned}
\frac{\operatorname{Pr}\left\{L^{\frac{1}{3}}(x) \in d t\right\}}{d t} & =-\frac{\partial}{\partial t} \int_{0}^{x} \frac{t}{s} \frac{1}{\sqrt[3]{3 s}} \operatorname{Ai}\left(\frac{t}{\sqrt[3]{3 s}}\right) d s \\
& =-\int_{0}^{x} \frac{1}{s \sqrt[3]{3 s}} \operatorname{Ai}\left(\frac{t}{\sqrt[3]{3 s}}\right) d s-\int_{0}^{x} \frac{t}{s \sqrt[3]{3 s}} \operatorname{Ai}^{\prime}\left(\frac{t}{\sqrt[3]{3 s}}\right) \frac{d s}{\sqrt[3]{3 s}} .
\end{aligned}
$$

Since

$$
\frac{\partial}{\partial s} \operatorname{Ai}\left(\frac{t}{\sqrt[3]{3 s}}\right)=-\frac{t}{3 s \sqrt[3]{3 s}} \operatorname{Ai}^{\prime}\left(\frac{t}{\sqrt[3]{3 s}}\right)
$$

we conclude that, for $x>0, t>0$,

$$
\begin{aligned}
l_{\frac{1}{3}}(t, x) & =\frac{\operatorname{Pr}\left\{L^{\frac{1}{3}}(x) \in d t\right\}}{d t} \\
& =\int_{0}^{x} \frac{-1}{s \sqrt[3]{3 s}} \operatorname{Ai}\left(\frac{t}{\sqrt[3]{3 s}}\right) d s+\int_{0}^{x} \frac{3}{\sqrt[3]{3 s}} \frac{\partial}{\partial s} \operatorname{Ai}\left(\frac{t}{\sqrt[3]{3 s}}\right) d s \\
& =\int_{0}^{x} \frac{-1}{s \sqrt[3]{3 s}} \operatorname{Ai}\left(\frac{t}{\sqrt[3]{3 s}}\right) d s+\left[\frac{3}{\sqrt[3]{3 s}} \operatorname{Ai}\left(\frac{t}{\sqrt[3]{3 s}}\right)\right]_{s=0}^{s=x}+\int_{0}^{x} \frac{d s}{s \sqrt[3]{3 s}} \operatorname{Ai}\left(\frac{t}{\sqrt[3]{3 s}}\right) \\
& =\frac{3}{\sqrt[3]{3 x}} \operatorname{Ai}\left(\frac{t}{\sqrt[3]{3 x}}\right) .
\end{aligned}
$$

In the last step we took into account the asymptotic expansion 7.2.19 of Bleistein and Handelsman [4].

With similar calculation we obtain the law $h_{\frac{2}{3}}(x, t)$ of the process $H^{\frac{2}{3}}(t), t>0$, which is expressed in terms of Airy function. From the general series expression of the stable law (see [16]) we have that,

$$
\begin{aligned}
& h_{\frac{2}{3}}(x, 1)= \\
= & \frac{2}{3 \pi} \sum_{k=0}^{\infty}(-1)^{k} \frac{\Gamma\left(\frac{2}{3}(k+1)\right)}{k !} x^{-\frac{2}{3}(k+1)-1} \sin \left(\frac{2 \pi}{3}(k+1)\right) \\
= & \frac{2}{3 \pi \sqrt{\pi}} \sum_{k=0}^{\infty} \frac{(-1)^{k}}{k !} \frac{x^{-\frac{2}{3}(k+1)-1}}{2^{1-\frac{2}{3}(k+1)}} \Gamma\left(\frac{k+1}{3}\right) \sin \left(\frac{2 \pi}{3}(k+1)\right) \int_{0}^{\infty} d w e^{-w} w^{\frac{k+1}{3}+\frac{1}{2}-1} \\
= & \frac{1}{x} \sqrt[3]{\frac{2^{2}}{3 x^{2}}} \frac{1}{\sqrt{\pi}} \int_{0}^{\infty} e^{-w} w^{-\frac{1}{6}} \mathrm{Ai}\left(-\sqrt[3]{\frac{2^{2} w}{3 x^{2}}}\right) d w
\end{aligned}
$$


and thus, in force of the fact that $H^{\frac{2}{3}}(t) \stackrel{\text { law }}{=} t^{\frac{3}{2}} H^{\frac{2}{3}}(1)$,

$$
h_{\frac{2}{3}}(x, t)=\frac{t}{\sqrt{\pi}} \frac{1}{x} \int_{0}^{\infty} d w e^{-w} w^{-\frac{1}{6}} \sqrt[3]{\frac{2^{2}}{3 x^{2}}} \mathrm{Ai}\left(-t \sqrt[3]{\frac{2^{2} w}{3 x^{2}}}\right) .
$$

Remark 4.4. We check that the distribution (4.58) integrates to unity. We have that

$$
\begin{aligned}
& \int_{0}^{\infty} h_{\frac{2}{3}}(x, t) d x= \\
&= \frac{t}{\sqrt{\pi}} \int_{0}^{\infty} d w e^{-w} w^{-\frac{1}{6}} \sqrt[3]{\frac{2^{2}}{3}} \int_{0}^{\infty} d x x^{-\frac{2}{3}-1} \mathrm{Ai}\left(-t \sqrt[3]{\frac{2^{2} w}{3 x^{2}}}\right) \\
& y=x^{-\frac{2}{3}} t \sqrt[3]{\frac{2^{2} w}{3}} \frac{t}{\sqrt{\pi}} \int_{0}^{\infty} d w e^{-w} w^{-\frac{1}{6}} \sqrt[3]{\frac{2^{2}}{3}} \frac{3}{2}\left(t \sqrt[3]{\frac{2^{2} w}{3}}\right)^{-1} \int_{0}^{\infty} d y \mathrm{Ai}(-y) \\
&=\frac{1}{\sqrt{\pi}} \int_{0}^{\infty} d w e^{-w} w^{-\frac{1}{6}} \sqrt[3]{\frac{2^{2}}{3}}\left(\sqrt[3]{\frac{2^{2} w}{3}}\right)^{-1} \sqrt{\sqrt{\pi}} \int_{0}^{\infty} d w e^{-w} w^{-\frac{1}{6}-\frac{1}{3}}=\frac{1}{\sqrt{\pi}} \int_{0}^{\infty} d w e^{-w} w^{\frac{1}{2}-1}=1
\end{aligned}
$$

where we used the fact that

$$
\int_{0}^{\infty} d y \operatorname{Ai}(-y)=\frac{2}{3}
$$

For the law of the process $L^{\frac{2}{3}}(x), x>0$, we therefore have that

$$
\begin{aligned}
\operatorname{Pr}\left\{L^{\frac{2}{3}}(x)<t\right\} & =\operatorname{Pr}\left\{H^{\frac{2}{3}}(t)>x\right\} \\
& =\int_{0}^{\infty} \int_{x}^{\infty} \frac{t}{\sqrt{\pi}} \frac{1}{z} \sqrt[3]{\frac{2^{2}}{3 z^{2}}} \mathrm{Ai}\left(-t \sqrt[3]{\frac{2^{2} w}{3 z^{2}}}\right) e^{-w} w^{-\frac{1}{6}} d w d z
\end{aligned}
$$

and thus

$$
\begin{aligned}
l_{\frac{2}{3}}(t, x)= & \int_{0}^{\infty} \int_{x}^{\infty} \frac{d w d z}{z \sqrt{\pi}} \sqrt[3]{\frac{2^{2}}{3 z^{2}}} \mathrm{Ai}\left(-t \sqrt[3]{\frac{2^{2} w}{3 z^{2}}}\right) e^{-w} w^{-\frac{1}{6}} \\
& -\int_{0}^{\infty} \int_{x}^{\infty} \frac{t}{z \sqrt{\pi}} \sqrt[3]{\frac{2^{2}}{3 z^{2}}} \sqrt[3]{\frac{2^{2} w}{3 z^{2}}} \mathrm{Ai}^{\prime}\left(-t \sqrt[3]{\frac{2^{2} w}{3 z^{2}}}\right) d z d w \\
= & \int_{0}^{\infty} \int_{x}^{\infty} \frac{d w d z}{z \sqrt{\pi}} \sqrt[3]{\frac{2^{2}}{3 z^{2}}} \mathrm{Ai}\left(-t \sqrt[3]{\frac{2^{2} w}{3 z^{2}}}\right) e^{-w} w^{-\frac{1}{6}} \\
& -\frac{3}{2} \int_{x}^{\infty} \int_{0}^{\infty} \frac{1}{\sqrt{\pi}} \sqrt[3]{\frac{2^{2}}{3 z^{2}}} e^{-w} w^{-\frac{1}{6}} \frac{\partial}{\partial z} \mathrm{Ai}\left(-t \sqrt[3]{\frac{2^{2} w}{3 z^{2}}}\right) d w d z \\
= & \int_{0}^{\infty} \int_{x}^{\infty} \frac{d w d z}{z \sqrt{\pi}} \sqrt[3]{\frac{2^{2}}{3 z^{2}}} \mathrm{Ai}\left(-t \sqrt[3]{\frac{2^{2} w}{3 z^{2}}}\right) e^{-w} w^{-\frac{1}{6}} \\
& -\left[\frac{3}{2 \sqrt{\pi}} \int_{0}^{\infty} d w \sqrt[3]{\frac{2^{2}}{3 z^{2}}} e^{-w} w^{-\frac{1}{6}} \mathrm{Ai}\left(-t \sqrt[3]{\frac{2^{2} w}{3 z^{2}}}\right)\right]_{z=x}^{z=\infty}
\end{aligned}
$$




$$
\begin{aligned}
& -\int_{0}^{\infty} \int_{x}^{\infty} \frac{d w d z}{z \sqrt{\pi}} \sqrt[3]{\frac{2^{2}}{3 z^{2}}} \operatorname{Ai}\left(-t \sqrt[3]{\frac{2^{2} w}{3 z^{2}}}\right) e^{-w} w^{-\frac{1}{6}} \\
= & \frac{3}{2 \sqrt{\pi}} \int_{0}^{\infty} \sqrt[3]{\frac{2^{2}}{3 x^{2}}} e^{-w} w^{-\frac{1}{6}} \mathrm{Ai}\left(-t \sqrt[3]{\frac{2^{2} w}{3 x^{2}}}\right) d w .
\end{aligned}
$$

For checking that (4.62) integrates to unity one can perform calculation similar to that of Remark 4.4.

Now we have all the information to get the distribution of the process $L^{\frac{1}{3}}(t)$, $t>0$, by means of formula (2.50). We have that

$$
\begin{aligned}
l_{\frac{1}{3}}(x, t)= & \frac{\operatorname{Pr}\left\{L^{\frac{1}{3}}(t) \in d x\right\}}{d x} \\
= & \int_{0}^{t} l_{\frac{2}{3}}(x, t-s) h_{\frac{1}{3}}(s, 2 \lambda x) d s+2 \lambda \int_{0}^{t} l_{\frac{1}{3}}(2 \lambda x, s) h_{\frac{2}{3}}(t-s, x) d s \\
= & \int_{0}^{t} d s\left[\frac{3}{2 \sqrt{\pi}} \int_{0}^{\infty} d w \sqrt[3]{\frac{2^{2}}{3(t-s)^{2}}} e^{-w} w^{-\frac{1}{6}} \mathrm{Ai}\left(-x \sqrt[3]{\frac{2^{2} w}{3(t-s)^{2}}}\right) d w\right] \\
& \cdot \frac{2 \lambda x}{s \sqrt[3]{3 s}} \mathrm{Ai}\left(\frac{2 \lambda x}{\sqrt[3]{3 s}}\right)+2 \lambda \int_{0}^{t} d s \frac{3}{\sqrt[3]{3 s}} \mathrm{Ai}\left(\frac{2 \lambda x}{\sqrt[3]{3 s}}\right) \cdot \\
& \cdot \frac{s}{\sqrt{\pi}(t-s)} \int_{0}^{\infty} d w e^{-w} w^{-\frac{1}{6}} \sqrt[3]{\frac{2^{2}}{3(t-s)^{2}}} \mathrm{Ai}\left(-x \sqrt[3]{\frac{2^{2} w}{3(t-s)^{2}}}\right) \\
= & \frac{2 \lambda}{\sqrt{\pi}} \int_{0}^{t} d s \int_{0}^{\infty} d w e^{-w} w^{-\frac{1}{6}} \mathrm{Ai}\left(-x \sqrt[3]{\frac{2^{2} w}{3(t-s)^{2}}}\right) \mathrm{Ai}\left(\frac{2 \lambda x}{\sqrt[3]{3 s}}\right) \\
& \cdot \frac{3}{\sqrt[3]{3 s}} \sqrt[3]{\frac{2^{2}}{3(t-s)^{2}}}\left[\frac{x}{2 s}+\frac{s}{t-s}\right]
\end{aligned}
$$

Result (4.63) permits us to write explicitly the solution of the fractional telegraph equation (1.4) for $\nu=\frac{1}{3}, \beta=1$ and $n=1$, as

$$
w_{\frac{1}{3}}^{1}(x, t)=\int_{0}^{\infty} \frac{e^{-\frac{x^{2}}{4 c^{2} s}}}{\sqrt{4 \pi c^{2} s}} C_{\frac{1}{3}}(s, t) d s, \quad x \in \mathbb{R}, t>0 .
$$

4.3. The planar case. Let us consider the planar process

$$
\boldsymbol{T}(t)=(X(t), Y(t)), \quad t>0,
$$

with infinite directions and finite velocity $c$, investigated in Orsingher and De Gregorio [15], which has probability law (see formula 1.2 therein)

$$
r(x, y, t)=\frac{\lambda}{2 \pi c} \frac{e^{-\lambda t+\frac{\lambda}{c} \sqrt{c^{2} t^{2}-\left(x^{2}+y^{2}\right)}}}{\sqrt{c^{2} t^{2}-\left(x^{2}+y^{2}\right)}}, \quad x^{2}+y^{2}<c^{2} t^{2}, t>0,
$$

which satisfies the telegraph equation

$$
\left(\frac{\partial^{2}}{\partial t^{2}}+2 \lambda \frac{\partial}{\partial t}\right) r(x, y, t)=c^{2}\left(\frac{\partial^{2}}{\partial x^{2}}+\frac{\partial^{2}}{\partial y^{2}}\right) r(x, y, t) .
$$

The distribution of $\boldsymbol{T}(t), t>0$, has a singular component uniformly distributed on the circle $\partial C_{c t}=\left\{(x, y) \in \mathbb{R}^{2}: x^{2}+y^{2}=c^{2} t^{2}\right\}$ with probability mass equal to 
$e^{-\lambda t}$. The process $\boldsymbol{T}(t), t>0$, describes a random motion where directions change at Poisson paced times and the orientation of each segment of the sample paths is uniform in $[0,2 \pi)$.

Let $q(x, y, t)$ be the distribution obtained by means of the composition of the process $\boldsymbol{T}(t)$ with a reflecting Brownian motion with law

$$
p_{|B|}(s, t)=\frac{e^{\frac{-s^{2}}{4 t}}}{\sqrt{\pi t}}, \quad t>0, s>0
$$

which satisfies the equation

$$
\frac{{ }^{C} \partial^{\frac{1}{2}}}{\partial t^{\frac{1}{2}}} p_{|B|}(s, t)=-\frac{\partial}{\partial s} p_{|B|}(s, t)
$$

and also

$$
\frac{\partial}{\partial t} p_{|B|}(s, t)=\frac{\partial^{2}}{\partial s^{2}} p_{|B|}(s, t)
$$

We have the following theorem.

Theorem 4.3. The law of the composition

$$
\boldsymbol{Q}(t)=\boldsymbol{T}(|B(t)|), \quad t>0
$$

written as

$$
q(x, y, t)=\int_{0}^{\infty} r(x, y, s) p_{|B|}(s, t) d s,
$$

satisfies the 2-dimensional time-fractional equation

$$
\left(\frac{\partial}{\partial t}+2 \lambda \frac{{ }^{C} \partial^{\frac{1}{2}}}{\partial t^{\frac{1}{2}}}\right) q(x, y, t)=c^{2}\left(\frac{\partial^{2}}{\partial x^{2}}+\frac{\partial^{2}}{\partial y^{2}}\right) q(x, y, t), \quad x, y \in \mathbb{R}, t>0
$$

subject to the initial condition $q(x, y, 0)=\delta(x, y)$.

Proof. By considering (4.72) and (4.69) we can write

$$
\begin{aligned}
\frac{{ }^{C} \partial^{\frac{1}{2}}}{\partial t^{\frac{1}{2}}} q(x, y, t) & =\int_{0}^{\infty} r(x, y, s) \frac{{ }^{C} \partial^{\frac{1}{2}}}{\partial t^{\frac{1}{2}}} p_{|B|}(s, t) d s \\
& =\int_{0}^{\infty} r(x, y, s)\left(-\frac{\partial}{\partial s} p_{|B|}(s, t)\right) d s \\
& =\left[-p_{|B|}(s, t) r(x, y, s)\right]_{s=0}^{s=\infty}+\int_{0}^{\infty} p_{|B|}(s, t) \frac{\partial}{\partial s} r(x, y, s) d s .
\end{aligned}
$$

In the previous step it must be taken into account that the boundary $\partial C_{c s}$ is excluded. From (4.72) and (4.70) we have that

$$
\begin{aligned}
\frac{\partial}{\partial t} q(x, y, t) & =\int_{0}^{\infty} r(x, y, s) \frac{\partial}{\partial t} p_{|B|}(s, t) d s=\int_{0}^{\infty} r(x, y, s) \frac{\partial^{2}}{\partial s^{2}} p_{|B|}(s, t) d s \\
& =\left[r(x, y, s) \frac{\partial}{\partial s} p_{|B|}(s, t)\right]_{s=0}^{s=\infty}-\int_{0}^{\infty} \frac{\partial}{\partial s} r(x, y, s) \frac{\partial}{\partial s} p_{|B|}(s, t) d s \\
& =-\left[p_{|B|}(s, t) \frac{\partial}{\partial s} r(x, y, s)\right]_{s=0}^{s=\infty}+\int_{0}^{\infty} p_{|B|}(s, t) \frac{\partial^{2}}{\partial s^{2}} r(x, y, s) d s
\end{aligned}
$$


Thus, by looking at (4.67), (4.74) and (4.75) we obtain

$$
\begin{aligned}
& \frac{\partial}{\partial t} q(x, y, t)+2 \lambda \frac{{ }^{C} \partial^{\frac{1}{2}}}{\partial t^{\frac{1}{2}}} q(x, y, t)= \\
= & \int_{0}^{\infty} p_{|B|}(s, t)\left[\frac{\partial^{2}}{\partial s^{2}} r(x, y, s)+2 \lambda \frac{\partial}{\partial s} r(x, y, s)\right] d s \\
= & \int_{0}^{\infty} p_{|B|}(s, t) c^{2}\left(\frac{\partial^{2}}{\partial x^{2}}+\frac{\partial^{2}}{\partial y^{2}}\right) r(x, y, s) d s=c^{2}\left(\frac{\partial^{2}}{\partial x^{2}}+\frac{\partial^{2}}{\partial y^{2}}\right) q(x, y, t) .
\end{aligned}
$$

which means that $q(x, y, t)$ satisfies equation (4.73).

It is easy to show that the process $\boldsymbol{Q}(t)=\boldsymbol{T}(|B(t)|), t>0$, has not the same law of the process $\boldsymbol{W}_{2}(t)=\boldsymbol{B}_{2}\left(c^{2} \mathcal{L}^{\frac{1}{2}}(t)\right), t>0$. However it is possible to construct a planar process, say $\boldsymbol{T}(t), t>0$ (which is a slightly different version of $\boldsymbol{T}(t), t>0$ ) composed with a suitable "time process" which has the same distribution as $\boldsymbol{W}_{2}(t)$, $t>0$. The planar random motion $\mathfrak{T}(t), t>0$, with distribution

$$
\mathfrak{r}(x, y, t)=\frac{\lambda e^{-\lambda t}}{2 \pi c}\left[\frac{e^{\frac{\lambda}{c} \sqrt{c^{2} t^{2}-\left(x^{2}+y^{2}\right)}}+e^{-\frac{\lambda}{c} \sqrt{c^{2} t^{2}-\left(x^{2}+y^{2}\right)}}}{\sqrt{c^{2} t^{2}-\left(x^{2}+y^{2}\right)}}\right],
$$

where $(x, y) \in C_{c t}=\left\{(x, y): x^{2}+y^{2}<c^{2} t^{2}\right\}$, can be constructed starting from the model dealt with in Orsingher and De Gregorio [15]. The distribution is based on the solution to the planar telegraph equation

$$
\left(\frac{\partial^{2}}{\partial t^{2}}+2 \lambda \frac{\partial}{\partial t}\right) \mathfrak{r}(x, y, t)=c^{2}\left(\frac{\partial^{2}}{\partial x^{2}}+\frac{\partial^{2}}{\partial y^{2}}\right) \mathfrak{r}(x, y, t)
$$

namely

$$
\mathfrak{r}(x, y, t)=\frac{e^{-\lambda t}}{\sqrt{c^{2} t^{2}-\left(x^{2}+y^{2}\right)}}\left[A e^{\frac{\lambda}{c} \sqrt{c^{2} t^{2}-\left(x^{2}+y^{2}\right)}}+B e^{-\frac{\lambda}{c} \sqrt{c^{2} t^{2}-\left(x^{2}+y^{2}\right)}}\right],
$$

with $A=B=\frac{\lambda}{2 \pi c}$ and thus we can easily check that

$$
\iint_{C_{c t}} d x d y \mathfrak{r}(x, y, t)=1-e^{-2 \lambda t} .
$$

We take a particle starting from the origin, moving at finite velocity $c$, and changing direction (chosen with uniform distribution) at Poisson times and neglect displacements started off by even-labelled times. The sample paths of this motion are constructed by piecing together only odd-order displacements of the planar motion $\boldsymbol{T}(t), t>0$. The process just described has distribution (4.77) as shown below

$$
\begin{aligned}
& \mathfrak{r}(x, y, t)= \\
= & \frac{\operatorname{Pr}\{\mathfrak{T}(t) \in d \boldsymbol{x}\}}{d \boldsymbol{x}}=\frac{\lambda e^{-\lambda t}}{2 \pi c}\left[\frac{e^{\frac{\lambda}{c} \sqrt{c^{2} t^{2}-\left(x^{2}+y^{2}\right)}}+e^{-\frac{\lambda}{c} \sqrt{c^{2} t^{2}-\left(x^{2}+y^{2}\right)}}}{\sqrt{c^{2} t^{2}-\left(x^{2}+y^{2}\right)}}\right] \\
= & \frac{\lambda^{2}}{c^{2}} \frac{1}{\pi} e^{-\lambda t}\left[\sum_{k=0}^{\infty}\left(\frac{\lambda}{c} \sqrt{c^{2} t^{2}-\left(x^{2}+y^{2}\right)}\right)^{2 k-1} \frac{1}{(2 k) !}\right] \\
= & \frac{\lambda^{2}}{c^{2}} \frac{1}{\pi} \sum_{k=0}^{\infty}\left(\frac{\lambda}{c}\right)^{2 k-1}(2 k+1)\left(c^{2} t^{2}-\left(x^{2}+y^{2}\right)\right)^{k-\frac{1}{2}} \frac{e^{-\lambda t}}{(2 k) !(2 k+1)} \frac{(\lambda t)^{2 k+1}}{(\lambda t)^{2 k+1}}
\end{aligned}
$$




$$
\begin{aligned}
& =2 \sum_{k=0}^{\infty} \operatorname{Pr}\{X(t) \in d x, Y(t) \in d y \mid N(t)=2 k+1\} e^{-\lambda t} \frac{(\lambda t)^{2 k+1}}{(2 k+1) !} \\
& =2 \sum_{k=0}^{\infty} \operatorname{Pr}\{\boldsymbol{T}(t) \in d \boldsymbol{x} \mid N(t)=2 k+1\} e^{-\lambda t} \frac{(\lambda t)^{2 k+1}}{(2 k+1) !}
\end{aligned}
$$

where, for $x^{2}+y^{2}<c^{2} t^{2}($ see $[15])$,

$$
\frac{\operatorname{Pr}\{X(t) \in d x, Y(t) \in d y \mid N(t)=n\}}{d x d y}=\frac{n}{2 n(c t)^{n}}\left(c^{2} t^{2}-\left(x^{2}+y^{2}\right)\right)^{\frac{n}{2}-1},
$$

and

$$
2 e^{-\lambda t} \sum_{k=0}^{\infty} \frac{(\lambda t)^{2 k+1}}{(2 k+1) !}=\sum_{k=0}^{\infty} 2 \operatorname{Pr}\{N(t)=2 k+1\}=1-e^{-2 \lambda t}
$$

The factor 2 appearing in (4.81) and (4.83) can be interpreted as follows. The displacements generated by an even number of Poisson events are disregarded and replaced by displacements produced by an odd number of deviations. Therefore, odd-order Poisson events ignite twice the displacements considered in (4.81).

Theorem 4.4. The composition with distribution

$$
\mathfrak{q}(x, y, t)=\int_{0}^{\infty} d s \mathfrak{r}(x, y, s)\left[p_{|B|}(s, t)+\frac{1}{2 \lambda} \frac{\partial^{\frac{1}{2}}}{\partial t^{\frac{1}{2}}} p_{|B|}(s, t)\right],
$$

which satisfies the time-fractional equation

$$
\left(\frac{\partial}{\partial t}+2 \lambda \frac{{ }^{C} \frac{1}{2}}{\partial t^{\frac{1}{2}}}\right) \mathfrak{q}(x, y, t)=c^{2}\left(\frac{\partial^{2}}{\partial x^{2}}+\frac{\partial^{2}}{\partial y^{2}}\right) \mathfrak{q}(x, y, t),
$$

has the same law of the process $\boldsymbol{W}_{2}(t)=\boldsymbol{B}_{2}\left(c^{2} L^{\frac{1}{2}}(t)\right)$.

Proof. We begin by evaluating the Fourier-Laplace transform of (4.84).

$$
\begin{aligned}
& \widehat{\widetilde{\mathfrak{q}}}(\xi, \alpha, \mu) \\
= & \int_{0}^{\infty} d s \int_{0}^{\infty} d t e^{-\mu t} \int_{C_{c t}} d x d y e^{i \xi x+i \alpha y} \mathfrak{r}(x, y, s)\left[p_{|B|}(s, t)+\frac{1}{2 \lambda} \frac{\partial^{\frac{1}{2}}}{\partial t^{\frac{1}{2}}} p_{|B|}(s, t)\right] \\
= & \frac{2 \lambda+\sqrt{\mu}}{2 \lambda \sqrt{\mu}} \int_{0}^{\infty} d s \int_{C_{c t}} d x d y e^{i \xi x+i \alpha y} \mathfrak{r}(x, y, s) e^{-s \sqrt{\mu}} .
\end{aligned}
$$

Now we need the Fourier transform of the law $\mathfrak{r}(x, y, t)$ of the process $\mathfrak{T}(t), t>0$, which reads

$$
\begin{aligned}
& \widehat{\mathfrak{r}}(\xi, \alpha, t)= \\
= & \frac{\lambda e^{-\lambda t}}{2 \pi c} \iint_{C_{c t}} e^{i \xi x+i \alpha y}\left[\frac{e^{\frac{\lambda}{c} \sqrt{c^{2} t^{2}-\left(x^{2}+y^{2}\right)}}+e^{-\frac{\lambda}{c} \sqrt{c^{2} t^{2}-\left(x^{2}+y^{2}\right)}}}{\sqrt{c^{2} t^{2}-\left(x^{2}+y^{2}\right)}}\right] d x d y \\
= & \frac{\lambda e^{-\lambda t}}{2 \pi c} \int_{0}^{2 \pi} d \theta \int_{0}^{c t} d \rho \rho e^{i \rho(\xi \cos \theta+\alpha \sin \theta)} \frac{\lambda}{c} \frac{e^{\frac{\lambda}{c} \sqrt{c^{2} t^{2}-\rho^{2}}}+e^{-\frac{\lambda}{c}} \sqrt{c^{2} t^{2}-\rho^{2}}}{\sqrt{c^{2} t^{2}-\rho^{2}}} \\
= & \frac{2 \lambda^{2} e^{-\lambda t}}{c^{2}} \int_{0}^{c t} \rho \sum_{m=0}^{\infty}\left(\frac{\lambda}{c} \sqrt{c^{2} t^{2}-\rho^{2}}\right)^{2 m-1} \frac{1}{(2 m) !} J_{0}\left(\rho \sqrt{\xi^{2}+\alpha^{2}}\right) d \rho
\end{aligned}
$$




$$
\begin{aligned}
& =\frac{2 \lambda e^{-\lambda t}}{c} \sum_{m=0}^{\infty} \frac{\left(\frac{\lambda}{c}\right)^{2 m}}{(2 m) !} \sum_{k=0}^{\infty} \frac{(-1)^{k}\left(\frac{\sqrt{\xi^{2}+\alpha^{2}}}{2}\right)^{2 k}}{(k !)^{2}} \cdot \int_{0}^{c t}\left(c^{2} t^{2}-\rho^{2}\right)^{m-\frac{1}{2}} \rho^{2 k+1} d \rho \\
& =\frac{2 \lambda e^{-\lambda t}}{c} \sum_{m=0}^{\infty} \frac{\left(\frac{\lambda}{c}\right)^{2 m}}{(2 m) !} \sum_{k=0}^{\infty} \frac{(-1)^{k}\left(\frac{\sqrt{\xi^{2}+\alpha^{2}}}{2}\right)^{2 k}}{2(k !)^{2}(c t)^{-(2 m+2 k+1)}} \int_{0}^{1} y^{k}(1-y)^{m-\frac{1}{2}} d y \\
& =\frac{\lambda}{c} e^{-\lambda t} \sum_{m=0}^{\infty}\left(\frac{\lambda}{c}\right)^{2 m} \frac{1}{(2 m) !} \sum_{k=0}^{\infty}(-1)^{k}\left(\frac{\sqrt{\xi^{2}+\alpha^{2}}}{2}\right)^{2 k} \frac{(c t)^{2 m+2 k+1} \Gamma\left(m+\frac{1}{2}\right)}{k ! \Gamma\left(k+m+1+\frac{1}{2}\right)} .
\end{aligned}
$$

Thus, from (4.86), we have that

$$
\widetilde{\mathfrak{\mathfrak { q }}}(\xi, \alpha, \mu)=\frac{2 \lambda+\sqrt{\mu}}{2 \lambda \sqrt{\mu}} \int_{0}^{\infty} d s \widehat{\mathfrak{r}}(\xi, \alpha, t) e^{-s \sqrt{\mu}}=\frac{1+2 \lambda \mu^{-\frac{1}{2}}}{\mu+2 \lambda \sqrt{\mu}+c^{2}\left(\xi^{2}+\alpha^{2}\right)}
$$

in force of the calculation

$$
\begin{aligned}
& \int_{0}^{\infty} d s \widehat{\mathfrak{r}}(\xi, \alpha, s) e^{-s \sqrt{\mu}}= \\
& =\frac{\lambda}{c} \int_{0}^{\infty} d s e^{-\lambda s} \sum_{m=0}^{\infty} \frac{\lambda^{2 m}}{c^{2 m}(2 m) !} \sum_{k=0}^{\infty} \frac{(-1)^{k}\left(\frac{\sqrt{\xi^{2}+\alpha^{2}}}{2}\right)^{2 k} \Gamma\left(m+\frac{1}{2}\right)}{k !(c s)^{-(2 m+2 k+1)} \Gamma\left(k+m+1+\frac{1}{2}\right)} e^{-s \sqrt{\mu}} \\
& =\lambda \sum_{m=0}^{\infty} \frac{\sqrt{\pi} 2^{1-2 m} \Gamma(2 m)}{\lambda^{-2 m}(2 m) ! \Gamma(m)} \sum_{k=0}^{\infty} \frac{(-1)^{k}\left(\frac{\sqrt{\xi^{2}+\alpha^{2}}}{2}\right)^{2 k} c^{2 k}}{k ! \Gamma\left(k+m+1+\frac{1}{2}\right)} \int_{0}^{\infty} e^{-s(\lambda+\sqrt{\mu})} s^{2 m+2 k+1} d s \\
& =\frac{\lambda}{2(\lambda+\sqrt{\mu})^{2}} \sum_{m=0}^{\infty} \frac{\lambda^{2 m} \sqrt{\pi} 2^{1-2 m}}{m !(\lambda+\sqrt{\mu})^{2 m}} \sum_{k=0}^{\infty} \frac{(-1)^{k}\left(\frac{\sqrt{\xi^{2}+\alpha^{2}}}{2}\right)^{2 k}}{k !(\lambda+\sqrt{\mu})^{2 k} c^{-2 k}} \frac{\Gamma(2 k+2 m+2)}{\Gamma\left(k+m+1+\frac{1}{2}\right)} \\
& =\frac{\sqrt{\pi} \lambda}{2(\lambda+\sqrt{\mu})^{2}} \sum_{m=0}^{\infty} \frac{\lambda^{2 m} 2^{1-2 m}}{m !(\lambda+\sqrt{\mu})^{2 m}} \sum_{k=0}^{\infty} \frac{(-1)^{k}\left(\frac{\sqrt{\xi^{2}+\alpha^{2}}}{2}\right)^{2 k}}{k !(\lambda+\sqrt{\mu})^{2 k} c^{-2 k}} \frac{\Gamma(k+m+1)}{2^{1-2(k+m+1)} \sqrt{\pi}} \\
& =\frac{2 \lambda}{(\lambda+\sqrt{\mu})^{2}} \sum_{m=0}^{\infty} \frac{\lambda^{2 m}}{m !(\lambda+\sqrt{\mu})^{2 m}} \sum_{k=0}^{\infty} \frac{(-1)^{k}\left(\sqrt{\xi^{2}+\alpha^{2}}\right)^{2 k}}{k !(\lambda+\sqrt{\mu})^{2 k} c^{-2 k}} \int_{0}^{\infty} e^{-u} u^{k+m} d u \\
& =\frac{2 \lambda}{(\lambda+\sqrt{\mu})^{2}} \int_{0}^{\infty} d u e^{u \frac{\lambda^{2}}{(\lambda+\sqrt{\mu})^{2}}-u \frac{c^{2}\left(\xi^{2}+\alpha^{2}\right)}{(\lambda+\sqrt{\mu})^{2}}-u}=\frac{\frac{2 \lambda}{(\lambda+\sqrt{\mu})^{2}}}{1-\frac{\lambda^{2}}{(\lambda+\sqrt{\mu})^{2}}+\frac{c^{2}\left(\xi^{2}+\alpha^{2}\right)}{(\lambda+\sqrt{\mu})^{2}}} \\
& =\frac{2 \lambda}{(\lambda+\sqrt{\mu})^{2}-\lambda^{2}+c^{2}\left(\xi^{2}+\alpha^{2}\right)}=\frac{2 \lambda}{\mu+2 \lambda \sqrt{\mu}+c^{2}\left(\xi^{2}+\alpha^{2}\right)} \text {. }
\end{aligned}
$$


The Fourier-Laplace transform of the law of the process $\boldsymbol{B}_{2}\left(c^{2} \mathcal{L}^{\frac{1}{2}}(t)\right)$ is written as in (4.15) for $n=2, \beta=1$ and $\nu=\frac{1}{2}$ as the following calculation shows

$$
\begin{aligned}
\widehat{w_{\frac{1}{2}}^{1}}(\xi, \alpha, t) & =\int_{0}^{\infty} \widehat{p_{\boldsymbol{B}}}\left(\xi, \alpha, c^{2} s\right) \widetilde{\mathbb{l}_{\frac{1}{2}}}(s, \mu) d s \\
& =\left(1+2 \lambda \mu^{-\frac{1}{2}}\right) \int_{0}^{\infty} e^{-\mu s-\left(\xi^{2}+\alpha^{2}\right) c^{2} s}\left[e^{-2 \lambda s \sqrt{\mu}}+2 \lambda \frac{e^{-2 \lambda s \sqrt{\mu}}}{\sqrt{\mu}}\right] d s \\
& =\frac{1+2 \lambda \mu^{-\frac{1}{2}}}{2 \lambda \sqrt{\mu}+\mu+c^{2}\left(\xi^{2}+\alpha^{2}\right)}
\end{aligned}
$$

In the previous calculation we use the Laplace transform of $l_{\frac{1}{2}}(x, t)$ obtained in (4.28). The proof is complete since (4.90), coincides with (4.88) and with the Fourier-Laplace transform of (4.85).

Remark 4.5. Since for the first passage time $\tau_{\frac{s}{\sqrt{2}}}=\inf \left\{z: B(z)=\frac{s}{\sqrt{2}}\right\}$ of a Brownian motion through level $\frac{s}{\sqrt{2}}$ we have that

$$
\int_{0}^{\infty} e^{-\mu t} \operatorname{Pr}\left\{\tau_{\frac{s}{\sqrt{2}}} \in d t\right\}=e^{-s \sqrt{\mu}}
$$

and

$$
\int_{0}^{\infty} e^{-\mu t} \frac{\partial^{\frac{1}{2}}}{\partial t^{\frac{1}{2}}} p_{|B|}(s, t) d t=e^{-s \sqrt{\mu}}
$$

we can write

$$
\begin{aligned}
& \int_{0}^{\infty} \mathfrak{r}(x, y, s) \frac{\partial^{\frac{1}{2}}}{\partial t^{\frac{1}{2}}} p_{|B|}(s, t) d s=\int_{0}^{\infty} \mathfrak{r}(x, y, s) \frac{s}{\sqrt{2}} \frac{e^{-\frac{s^{2}}{4 t}}}{\sqrt{2 \pi t^{3}}} d s \\
= & \int_{0}^{\infty} \frac{\partial}{\partial s} \mathfrak{r}(x, y, s) \frac{e^{-\frac{s^{2}}{4 t}}}{\sqrt{\pi t}} d s=\int_{0}^{\infty} \frac{\partial}{\partial s} \mathfrak{r}(x, y, s) p_{|B|}(s, t) d s .
\end{aligned}
$$

This representation of the second term of (4.84) is extremely interesting because by integrating (4.93) in $C_{c t}$ we get

$$
\int_{0}^{\infty} \frac{\partial}{\partial s}\left(1-e^{-2 \lambda s}\right) p_{|B|}(s, t) d s=2 \lambda \int_{0}^{\infty} e^{-2 \lambda s} p_{|B|}(s, t) d s
$$

and yields the missing probability of the first term of (4.84).

Remark 4.6. We check that the law

$$
\mathfrak{q}(x, y, t)=\int_{0}^{\infty} \mathfrak{r}(x, y, s)\left[p_{|B|}(s, t)+\frac{1}{2 \lambda} \frac{\partial^{\frac{1}{2}}}{\partial t^{\frac{1}{2}}} p_{|B|}(s, t)\right] d s
$$

integrates to unity. By taking the $t$-Laplace transform, the integral with respect to $(x, y)$ becomes

$$
\begin{aligned}
& \iint_{C_{c t}} d x d y \int_{0}^{\infty} d t e^{-\mu t} \mathfrak{q}(x, y, t) \\
= & \int_{0}^{\infty}\left(1-e^{-2 \lambda s}\right)\left[\int_{0}^{\infty} e^{-\mu t}\left(p_{|B|}(s, t)+\frac{1}{2 \lambda} \frac{\partial^{\frac{1}{2}}}{\partial t^{\frac{1}{2}}} p_{|B|}(s, t)\right) d t\right] d s \\
= & \int_{0}^{\infty}\left(1-e^{-2 \lambda s}\right)\left[\frac{e^{-s \sqrt{\mu}}}{\sqrt{\mu}}+\frac{e^{-s \sqrt{\mu}}}{2 \lambda}\right] d s
\end{aligned}
$$




$$
\begin{aligned}
& =\left(\frac{1}{\sqrt{\mu}}+\frac{1}{2 \lambda}\right)\left[\int_{0}^{\infty} e^{-s \sqrt{\mu}} d s-\int_{0}^{\infty} e^{-s(2 \lambda+\sqrt{\mu})} d s\right] \\
& =\frac{2 \lambda+\sqrt{\mu}}{2 \lambda \sqrt{\mu}}\left(\frac{1}{\sqrt{\mu}}-\frac{1}{2 \lambda+\sqrt{\mu}}\right)=\frac{1}{\mu}=\int_{0}^{\infty} e^{-\mu t} d t .
\end{aligned}
$$

The same check can be done directly by taking into account formulas (4.93) and (4.94).

Relationships similar to $B\left(c^{2} \mathcal{L}^{\frac{1}{2}}(t)\right) \stackrel{\text { law }}{=} T(|B(t)|), t>0$, and the analogous one in the plane, cannot be established in spaces of dimension $n \geq 3$, because random motions governed by telegraph equations in such spaces have not been constructed. Random flights in $\mathbb{R}^{n}$ have been studied (Orsingher and De Gregorio [15]) but their distributions are not related to higher-dimensional telegraph equations.

\section{REFERENCES}

[1] H. Allouba and E. Nane. Interacting time-fractional and $\Delta^{\nu}$ PDE's systems via Brownian-time and Inverse-stable-Lévy-time Brownian sheets. Stochastics and Dynamics, 13(1): 1250012, 2013.

[2] H. Allouba and W. Zheng. Brownian-time processes: The PDE connection and the half-derivative generator. Ann. Probab., 29:1780 - 1795, 2001.

[3] A.V. Balakrishnan. Fractional powers of closed operators and semigroups generated by them. Pacific J. Math., 10:419 - 437, 1960.

[4] N. Bleistein and R.A. Handelsman. Asymptotic Expansions of Integrals. Dover Publications, 1986.

[5] S. Bochner. Diffusion equation and stochastic processes. Proc. Nat. Acad. Sciences, U.S.A., 35:368 - 370, 1949.

[6] R.D. DeBlassie. Iterated Brownian motion in an open set. Ann. Appl. Probab., 14: $1529-1558,2004$.

[7] W. Feller. On a generalization of Marcel Riesz' potentials and the semigroups generated by them. Comm. Sém. Math. Univ. Lund [Medd. Lunds Univ. Mat. Sem.], Tome Supplementaire, 1952.

[8] S.K. Foong and S. Kanno. Properties of the telegrapher's random process with or without a trap. Stoch. Proc. Appl., 53(1):147 - 173, 1994.

[9] Y. Fujita. Integrodifferential equation which interpolates the heat equation and the wave equation.I-II. Osaka J. Math, I: 27(2):797 - 804, 1990. II: 27(4):309 $-321,1990$.

[10] H.J. Haubold, A.M. Mathai and R.K. Saxena. Mittag-Leffler Functions and Their Applications. J. Appl. Math, 51 pages, 2011.

[11] A.A. Kilbas, H.M. Srivastava and J.J. Trujillo. Theory and Applications of Fractional Differential Equations. North-Holland Mathematics Studies, 204. Elsevier Science B.V., 2006.

[12] S. Momani. Analytic and approximate solutions of the space- and timefractional telegraph equations. Appl. Math. Comput., 170(2): 1126-1134, 2005.

[13] E. Orsingher and L. Beghin. Time-fractional telegraph equations and telegraph processes with Brownian time. Probab. Theory Related Fields, 128(1):141-160, 2004

[14] E. Orsingher and X. Zhao. The space-fractional telegraph equation and the related fractional telegraph process. Chin. Ann. Math., 24B(1):1-12, 2003. 
[15] E. Orsingher and A. De Gregorio. Random flights in higher spaces. J. Theoret. Probab., 20(4): 769 - 806, 2007.

[16] E. Orsingher and L. Beghin. Fractional diffusion equations and processes with randomly varying time. Ann. Probab., 37:206 - 249, 2009.

[17] A.I. Saichev and G.M. Zaslavsky. Fractional kinetic equations: Solutions and applications. Chaos, 7: $753-764,1997$.

[18] R.K. Saxena, A.M. Mathai and H.J. Haubold. Reaction-Diffusion Systems and Nonlinear Waves. Astrophys. Space Sci., 305:297 - 303, 2006.

[19] W. Stadje and S. Zacks. Telegraph processes with random velocities. J. Appl. Prob., 41:665 - 678, 2004.

[20] V.M. Zolotarev. One-dimensional stable distributions, volume 65 of Translations of Mathematical Monographs. American Mathematical Society, 1986. ISBN 0-8218-4519-5. Translated from the Russian by H. H. McFaden, Translation edited by Ben Silver.

Department of basic and applied sciences for engineers, SAPienza University of Rome

E-mail address: mirko.dovidio@uniroma1

Department of Statistical Sciences, Sapienza University of Rome

E-mail address: enzo.orsingher@uniroma1.it

Department of Statistical Sciences, Sapienza University of Rome

E-mail address: bruno.toaldo@uniroma1.it 